\title{
DIFFUSION LIMIT FOR THE RADIATIVE TRANSFER EQUATION PERTURBED BY A MARKOVIAN PROCESS
}

\author{
A. Debussche* ${ }^{*}$ S. De Moor ${ }^{*}$ and J. Vovelle ${ }^{\dagger}$
}

\begin{abstract}
We study the stochastic diffusive limit of a kinetic radiative transfer equation, which is non linear, involving a small parameter and perturbed by a smooth random term. Under an appropriate scaling for the small parameter, using a generalization of the perturbed test-functions method, we show the convergence in law to a stochastic non linear fluid limit.
\end{abstract}

Keywords: Kinetic equations, non-linear, diffusion limit, stochastic partial differential equations, perturbed test functions, Rosseland approximation, radiative transfer.

\section{Introduction}

In this paper, we are interested in the following non-linear equation

$$
\left\{\begin{array}{l}
\partial_{t} f^{\varepsilon}+\frac{1}{\varepsilon} a(v) \cdot \nabla_{x} f^{\varepsilon}=\frac{1}{\varepsilon^{2}} \sigma\left(\overline{f^{\varepsilon}}\right) L\left(f^{\varepsilon}\right)+\frac{1}{\varepsilon} f^{\varepsilon} m^{\varepsilon}, \\
f^{\varepsilon}(0)=f_{0}^{\varepsilon}, \quad t \in[0, T], x \in \mathbb{T}^{N}, v \in V .
\end{array}\right.
$$

where $(V, \mu)$ is a measured space, $a: V \rightarrow \mathbb{R}^{N}, \sigma: \mathbb{R} \rightarrow \mathbb{R}$. The notation $\bar{f}$ stands for the average over the velocity space $V$ of the function $f$, that is

$$
\bar{f}=\int_{V} f \mathrm{~d} \mu(v) .
$$

The operator $L$ is a linear operator of relaxation which acts on the velocity variable $v \in V$ only. It is given by

$$
L(f):=\bar{f} F-f,
$$

where $v \mapsto F(v)$ is a velocity equilibrium function such that

$$
F>0 \text { a.s., } \quad \bar{F}=1, \quad \sup _{v \in V} F(v)<\infty .
$$

The term $m^{\varepsilon}$ is a random process depending on $(t, x) \in \mathbb{R}^{+} \times \mathbb{R}^{N}$ (see section 2.2). The precise description of the problem setting will be given in the next section. In this paper, we study the behaviour in the limit $\varepsilon \rightarrow 0$ of the solution $f^{\varepsilon}$ of (1.1).

Concerning the physical background in the deterministic case $\left(m^{\varepsilon} \equiv 0\right)$, equation (1.1) describes the interaction between a surrounding continuous medium and a flux of photons radiating through it in the absence of hydrodynamical motion. The unknown $f^{\varepsilon}(t, x, v)$ then stands for a distribution function of photons having position $x$ and velocity $v$ at time $t$. The

\footnotetext{
*IRMAR, ENS Rennes, CNRS, UEB. av Robert Schuman, F-35170 Bruz, France. Email: arnaud.debussche@ens-rennes.fr; sylvain.demoor@ens-rennes.fr

†Université de Lyon ; CNRS ; Université Lyon 1, Institut Camille Jordan, 43 boulevard du 11 novembre 1918, F-69622 Villeurbane Cedex, France. Email: vovelle@math.univ-lyon1.fr
} 
function $\sigma$ is the opacity of the matter. When the surrounding medium becomes very large compared to the mean free paths $\varepsilon$ of photons, the solution $f^{\varepsilon}$ to (1.1) is known to behave like $\rho F$ where $\rho$ is the solution of the Rosseland equation

$$
\partial_{t} \rho-\operatorname{div}_{x}\left(\sigma(\rho)^{-1} K \nabla_{x} \rho\right)=0, \quad(t, x) \in[0, T] \times \mathbb{T}^{N},
$$

and $F$ is the velocity equilibrium defined above. This is what we call the Rosseland approximation. In this paper, we investigate such an approximation where we have perturbed the deterministic equation by a smooth multiplicative random noise. To do so, we use the method of perturbed test-functions. This method provides an elegant way of deriving stochastic diffusive limit from random kinetic systems; it was first introduced by Papanicolaou, Stroock and Varadhan [11]. The book of Fouque, Garnier, Papanicolaou and Solna [9] presents many applications to this method. A generalization in infinite dimension of the perturbed test-functions method arose in recent papers of Debussche and Vovelle [7] and de Bouard and Gazeau [6].

In the deterministic case (that is when $m^{\varepsilon} \equiv 0$ ), the Rosseland approximation has been widely studied. In the paper of Bardos, Golse and Perthame [1], they derive the Rosseland approximation on a slightly more general equation of radiative transfer type than (1.1) where the solution also depends on the frequency variable $\nu$. Using the so-called Hilbert's expansion method, they prove a strong convergence of the solution of the radiative transfer equation to the solution of the Rosseland equation. In [2], the Rosseland approximation is proved in a weaker sense with weakened hypothesis on the various parameters of the radiative transfer equation, in particular on the opacity function $\sigma$.

In the stochastic setting, the case where $\sigma \equiv \sigma_{0}$ is constant has been studied in the paper of Debussche and Vovelle [7] where they prove the convergence in law of the solution of (1.1) to a limit stochastic fluid equation by mean of a generalization of the perturbed test-functions method. Thus the radiative transfer equation (1.1) is a first step in studying approximation diffusion on non-linear stochastic kinetic equations since the operator $\sigma(\bar{f}) L f$ stands for a simple non-linear perturbation of the classical linear relaxation operator $L$.

As expected, we have to handle some difficulties caused by this non-linearity. In the paper of Debussche and Vovelle [7] is proved the tightness of the family of processes $\left(\rho^{\varepsilon}\right)_{\varepsilon>0}$ in the space of time-continuous function with values in some negative Sobolev space $H^{-\eta}\left(\mathbb{T}^{N}\right)$. In our non-linear setting, this is not any more sufficient to succeed in passing to the limit as $\varepsilon$ goes to 0 . As a consequence, the main step to overcome this difficulty is to prove the tightness of the family of processes $\left(\rho^{\varepsilon}\right)_{\varepsilon>0}$ in the space $L^{2}\left(0, T ; L^{2}\left(\mathbb{T}^{N}\right)\right)$. This is made using averaging lemmas in the $L^{2}$ setting with a slight adaptation to our stochastic context. The main results about deterministic averaging lemmas that we will use in the sequel can be found in the paper of Jabin [10]. We point out that, thanks to this additional tightness result, we could handle the case of a more general and non-linear noise term in (1.1) of the form $\frac{1}{\varepsilon} m^{\varepsilon} \lambda\left(\overline{f^{\varepsilon}}\right) f^{\varepsilon}$ where $\lambda: \mathbb{R} \rightarrow \mathbb{R}$ is a bounded and continuous function. In particular, this remains valid in the linear case $\sigma \equiv 1$ studied in the paper [7] of Debussche and Vovelle so that this paper can provide some improvements to their result.

Aknowledgements: This work is partially supported by the french government thanks to the ANR program Stosymap. It also benefit from the support of the french government "Investissements d'Avenir" program ANR-11-LABX-0020-01. 


\section{Preliminaries and main result}

\subsection{Notations and hypothesis}

Let us now introduce the precise setting of equation (1.1). We work on a finite-time interval $[0, T]$ where $T>0$ and consider periodic boundary conditions for the space variable: $x \in \mathbb{T}^{N}$ where $\mathbb{T}^{N}$ is the $N$-dimensional torus. Regarding the velocity space $V$, we assume that $(V, \mu)$ is a measured space.

In the sequel, $L_{F^{-1}}^{2}$ denotes the $F^{-1}$ weighted $L^{2}\left(\mathbb{T}^{N} \times V\right)$ space equipped with the norm

$$
\|f\|^{2}:=\int_{\mathbb{T}^{N}} \int_{V} \frac{|f(x, v)|^{2}}{F(v)} \mathrm{d} \mu(v) \mathrm{d} x .
$$

We denote its scalar product by $(.,$.$) . We also need to work in the space L^{2}\left(\mathbb{T}^{N}\right)$, which will be often written $L^{2}$ for short when the context is clear. In what follows, we will often use the inequality

$$
\|\bar{f}\|_{L_{x}^{2}} \leq\|f\|,
$$

which is just Cauchy-Schwarz inequality and the fact that $\bar{F}=1$. We also introduce the Sobolev spaces on the torus $H^{\gamma}\left(\mathbb{T}^{N}\right)$, or $H^{\gamma}$ for short. For $\gamma \in \mathbb{N}$, they consist of periodic functions which are in $L^{2}\left(\mathbb{T}^{N}\right)$ as well as their derivatives up to order $\gamma$. For general $\gamma \geq 0$, they are easily defined by Fourier series. For $\gamma<0, H^{\gamma}\left(\mathbb{T}^{N}\right)$ is the dual of $H^{-\gamma}\left(\mathbb{T}^{N}\right)$.

Concerning the velocity mapping $a: V \rightarrow \mathbb{R}^{N}$, we shall assume that it is bounded, that is

$$
\sup _{v \in V}|a(v)|<\infty
$$

Furthermore, we suppose that the following null flux hypothesis holds

$$
\int_{V} a(v) F(v) \mathrm{d} \mu(v)=0
$$

and that the following matrix

$$
K:=\int_{V} a(v) \otimes a(v) F(v) \mathrm{d} \mu(v)
$$

is definite positive. Finally, to obtain some compactness in the space variable by means of averaging lemmas, we also assume the following standard condition:

$$
\forall \varepsilon>0, \forall(\xi, \alpha) \in S^{N-1} \times \mathbb{R}, \quad \mu(\{v \in V,|a(v) \cdot \xi+\alpha|<\varepsilon\}) \leq \varepsilon^{\theta},
$$

for some $\theta \in(0,1]$.

Let us now give several hypothesis on the opacity function $\sigma: \mathbb{R} \rightarrow \mathbb{R}$. We assume that

(H1) There exist two positive constants $\sigma_{*}, \sigma^{*}>0$ such that for almost all $x \in \mathbb{R}$, we have

$$
\sigma_{*} \leq \sigma(x) \leq \sigma^{*}
$$

(H2) the function $\sigma$ is Lipschitz continuous. 
Similarly as in the deterministic case, we expect with $(1.1)$ that $\sigma\left(\overline{f^{\varepsilon}}\right) L\left(f^{\varepsilon}\right)$ tends to zero with $\varepsilon$, so that we should determine the equilibrium of the operator $\sigma(\cdot) L(\cdot)$. In this case, since $\sigma>0$, they are clearly constituted by the functions of the form $\rho F$ with $\rho$ being independent of $v \in V$. Note that it can easily be seen that $\left.\sigma()^{-}\right) L(\cdot)$ is a bounded operator from $L_{F^{-1}}^{2}$ to $L_{F^{-1}}^{2}$ and that it is dissipative; precisely, for $f \in L_{F^{-1}}^{2}$,

$$
(\sigma(\bar{f}) L f, f)=-\left\|\sigma^{\frac{1}{2}}(\bar{f}) L f\right\|^{2} \leq 0 .
$$

In the sequel, we denote by $g(t, \cdot)$ the semi-group generated by the operator $\sigma(\cdot) L(\cdot)$ on $L_{F^{-1}}^{2}$. It verifies, for $f \in L_{F^{-1}}^{2}$,

$$
\left\{\begin{aligned}
\frac{d}{d t} g(t, f) & =\sigma(\overline{g(t, f)}) L g(t, f), \\
g(0, f) & =f,
\end{aligned}\right.
$$

and we can show that it is given by

$$
g(t, f)=\bar{f} F+(f-\bar{f} F) e^{-t \sigma(\bar{f})}, \quad t \geq 0, f \in L_{F^{-1}}^{2} .
$$

With the hypothesis (H1) made on $\sigma$, we deduce the following relaxation property of the operator $\sigma(\cdot) L(\cdot)$

$$
g(t, f) \longrightarrow \bar{f} F, \quad t \rightarrow \infty, \quad \text { in } L_{F^{-1}}^{2} .
$$

\subsection{The random perturbation}

The random term $m^{\varepsilon}$ is defined by

$$
m^{\varepsilon}(t, x):=m\left(\frac{t}{\varepsilon^{2}}, x\right),
$$

where $m$ is a stationary process on a probability space $(\Omega, \mathcal{F}, \mathbb{P})$ and is adapted to a filtration $\left(\mathcal{F}_{t}\right)_{t \geq 0}$. Note that $m^{\varepsilon}$ is adapted to the filtration $\left(\mathcal{F}_{t}^{\varepsilon}\right)_{t \geq 0}=\left(\mathcal{F}_{\varepsilon^{-2} t}\right)_{t \geq 0}$.

We assume that, considered as a random process with values in a space of spatially dependent functions, $m$ is a stationary homogeneous Markov process taking values in a subset $E$ of $W^{1, \infty}\left(\mathbb{T}^{N}\right)$. In the sequel, $E$ will be endowed with the norm $\|\cdot\|_{\infty}$ of $L^{\infty}\left(\mathbb{T}^{N}\right)$. Besides, we denote by $\mathcal{B}(E)$ the set of bounded functions from $E$ to $\mathbb{R}$ endowed with the norm $\|g\|_{\infty}:=$ $\sup _{n \in E}|g(n)|$ for $g \in \mathcal{B}(E)$.

We assume that $m$ is stochastically continuous. Note that $m$ is supposed not to depend on the variable $v$. For all $t \geq 0$, the law $\nu$ of $m_{t}$ is supposed to be centered

$$
\mathbb{E} m_{t}=\int_{E} n \mathrm{~d} \nu(n)=0 .
$$

We denote by $e^{t M}$ a transition semi-group on $E$ associated to $m$ and by $M$ its infinitesimal generator. $\mathrm{D}(M)$ stands for the domain of $M$; it is defined as follows:

$$
\mathrm{D}(M):=\left\{u \in \mathcal{B}(E), \lim _{h \rightarrow 0} \frac{e^{h M}-I}{h} u \text { exists in } \mathcal{B}(E)\right\},
$$


and if $u \in \mathrm{D}(M)$, we have

$$
M u:=\lim _{h \rightarrow 0} \frac{e^{h M}-I}{h} u \text { in } \mathcal{B}(E) .
$$

Moreover, we suppose that $m$ is ergodic and satisfies some mixing properties in the sense that there exists a subspace $\mathscr{P}_{M}$ of $\mathcal{B}(E)$ such that for any $g \in \mathscr{P}_{M}$, the Poisson equation

$$
M \psi=g-\int_{E} g(n) \mathrm{d} \nu(n)=: \widehat{g},
$$

has a unique solution $\psi \in \mathrm{D}(M)$ satisfying $\int_{E} \psi(n) d \nu(n)=0$. We denote by $M^{-1} \widehat{g}$ this unique solution, and assume that it is given by

$$
M^{-1} \widehat{g}(n)=-\int_{0}^{\infty} e^{t M} \widehat{g}(n) \mathrm{d} t, \quad n \in E .
$$

In particular, we suppose that the above integral is well defined. We need that $\mathscr{P}_{M}$ contains sufficiently many functions. Thus we assume that for all $f, g \in L_{F^{-1}}^{2}$, we have

$$
\psi_{f, g}^{(1)}: n \mapsto(f n, g) \in \mathscr{P}_{M},
$$

and we then define $M^{-1} I$ from $E$ into $W^{1, \infty}\left(\mathbb{T}^{N}\right)$ by

$$
\left(f M^{-1} I(n), g\right):=M^{-1} \psi_{f, g}^{(1)}(n), \quad \forall f, g \in L_{F^{-1}}^{2} .
$$

Then, we also suppose that for all $f, g, h \in L_{F^{-1}}^{2}$ and all continuous operator $B$ from $L_{F^{-1}}^{2}$ to the space of the continuous bilinear operators on $L_{F^{-1}}^{2} \times L_{F^{-1}}^{2}$,

$$
\psi_{f, g}^{(2)}: n \mapsto\left(f n M^{-1} I(n), g\right), \quad \psi_{B, f, g, h}^{(3)}: n \mapsto B(f)\left(g n, h M^{-1} I(n)\right) \in \mathscr{P}_{M} .
$$

We need a uniform bound in $W^{1, \infty}\left(\mathbb{T}^{N}\right)$ of all the functions of the variable $n \in E$ introduced above. Namely, we assume, for all $f, g \in L_{F^{-1}}^{2}$ and all continuous operator $B$ on $L_{F^{-1}}^{2}$,

$$
\begin{array}{ll}
\|n\|_{W^{1, \infty}\left(\mathbb{T}^{N}\right)} \leq C_{*}, & \left\|M^{-1} I(n)\right\|_{W^{1, \infty}\left(\mathbb{T}^{N}\right)} \leq C_{*}, \\
\left|M^{-1} \psi_{f, g}^{(2)}\right| \leq C_{*}\|f\|\|g\|, & \left|M^{-1} \psi_{B, f, g}^{(3)}\right| \leq C_{*}\|B(f)\|\|f\|\|g\| .
\end{array}
$$

Finally, we suppose that for all $f, g \in L_{F^{-1}}^{2}$,

$$
n \mapsto\left(f M^{-1} I(n), g\right)^{2} \in \mathrm{D}(M) \text { with }\left|M\left[\left(f M^{-1} I(n), g\right)^{2}\right]\right| \leq C_{*}\|f\|^{2}\|g\|^{2} .
$$

To describe the limiting stochastic partial differential equation, we then set

$$
k(x, y)=\mathbb{E} \int_{\mathbb{R}} m_{0}(y) m_{t}(x) d t, \quad x, y \in \mathbb{T}^{N} .
$$

We can easily show that the kernel $k$ belong to $L^{\infty}\left(\mathbb{T}^{N} \times \mathbb{T}^{N}\right)$ and, $m$ being stationary, that it is symmetric (see [7]). As a result, we introduce the operator $Q$ on $L^{2}\left(\mathbb{T}^{N}\right)$ associated to the kernel $k$

$$
Q f(x)=\int_{\mathbb{T}^{N}} k(x, y) f(y) \mathrm{d} y,
$$

which is self-adjoint, compact and non-negative (see [7]). As a consequence, we can define the square root $Q^{\frac{1}{2}}$ which is Hilbert-Schmidt on $L^{2}\left(\mathbb{T}^{N}\right)$.

Remark The above assumptions on the process $m$ are verified, for instance, when $m$ is a Poisson process taking values in a bounded subset $E$ of $W^{1, \infty}\left(\mathbb{T}^{N}\right)$. 


\subsection{Resolution of the kinetic equation}

In this section, we solve the linear evolution problem (1.1) thanks to a semi-group approach. We thus introduce the linear operator $A:=a(v) \cdot \nabla_{x}$ on $L_{F^{-1}}^{2}$ with domain

$$
\mathrm{D}(A):=\left\{f \in L_{F^{-1}}^{2}, \nabla_{x} f \in L_{F^{-1}}^{2}\right\} .
$$

The operator $A$ has dense domain and, since it is skew-adjoint, it is $m$-dissipative. Consequently $A$ generates a contraction semigroup $(\mathcal{T}(t))_{t \geq 0}$ (see [4]). We recall that $\mathrm{D}(A)$ is endowed with the norm $\|\cdot\|_{\mathrm{D}(A)}:=\|\cdot\|+\|A \cdot\|$, and that it is a Banach space.

Proposition 2.1. Let $T>0$ and $f_{0}^{\varepsilon} \in L_{F^{-1}}^{2}$. Then there exists a unique mild solution of (1.1) on $[0, T]$ in $L^{\infty}(\Omega)$, that is there exists a unique $f^{\varepsilon} \in L^{\infty}\left(\Omega, C\left([0, T], L_{F^{-1}}^{2}\right)\right)$ such that $\mathbb{P}-$ a.s.

$$
f_{t}^{\varepsilon}=\mathcal{T}\left(\frac{t}{\varepsilon}\right) f_{0}^{\varepsilon}+\int_{0}^{t} \mathcal{T}\left(\frac{t-s}{\varepsilon}\right)\left(\frac{1}{\varepsilon^{2}} \sigma\left(\overline{f_{s}^{\varepsilon}}\right) L f_{s}^{\varepsilon}+\frac{1}{\varepsilon} m_{s}^{\varepsilon} f_{s}^{\varepsilon}\right) d s, \quad t \in[0, T] .
$$

Assume further that $f_{0}^{\varepsilon} \in \mathrm{D}(A)$, then there exists a unique strong solution $f^{\varepsilon}$ which belongs to the spaces $L^{\infty}\left(\Omega, C^{1}\left([0, T], L_{F^{-1}}^{2}\right)\right)$ and $L^{\infty}(\Omega, C([0, T], \mathrm{D}(A)))$ of $(1.1)$.

Proof. Subsections 4.3.1 and 4.3.3 in [4] gives that $\mathbb{P}$-a.s. there exists a unique mild solution $f^{\varepsilon} \in C\left([0, T], L_{F^{-1}}^{2}\right)$ and it is not difficult to slightly modify the proof to obtain that in fact $f^{\varepsilon} \in L^{\infty}\left(\Omega, C\left([0, T], L_{F^{-1}}^{2}\right)\right.$ ) (we intensively use that for all $t \geq 0$ and $\varepsilon>0,\left\|m_{t}^{\varepsilon}\right\|_{W^{1, \infty}\left(\mathbb{T}^{N}\right)} \leq$ $\left.C_{*}\right)$.

Similarly, subsections 4.3 .1 and 4.3 .3 in [4] gives us $\mathbb{P}$-a.s. a strong solution $f^{\varepsilon}$ in the spaces $C^{1}\left([0, T], L_{F^{-1}}^{2}\right)$ and $C([0, T], \mathrm{D}(A))$ of $(1.1)$ and once again one can easily get that in fact $f^{\varepsilon}$ belongs to the spaces $L^{\infty}\left(\Omega, C^{1}\left([0, T], L_{F^{-1}}^{2}\right)\right)$ and $L^{\infty}(\Omega, C([0, T], \mathrm{D}(A)))$.

Remark If $f_{0}^{\varepsilon} \in \mathrm{D}(A)$, we thus have, for $\varepsilon>0$ fixed,

$$
\sup _{t \in[0, T]}\left\|f_{t}^{\varepsilon}\right\|+\sup _{t \in[0, T]}\left\|A f_{t}^{\varepsilon}\right\| \in L^{\infty}(\Omega) .
$$

\subsection{Main result}

We are now ready to state our main result.

Theorem 2.2. Assume that $\left(f_{0}^{\varepsilon}\right)_{\varepsilon>0}$ is bounded in $L_{F^{-1}}^{2}$ and that

$$
\rho_{0}^{\varepsilon}:=\int_{V} f_{0}^{\varepsilon} \mathrm{d} \mu(v) \underset{\varepsilon \rightarrow 0}{\longrightarrow} \rho_{0} \text { in } L^{2}\left(\mathbb{T}^{N}\right) .
$$

Then, for all $\eta>0$ and $T>0, \rho^{\varepsilon}:=\overline{f^{\varepsilon}}$ converges in law in $C\left([0, T], H^{-\eta}\left(\mathbb{T}^{N}\right)\right)$ and $L^{2}\left(0, T ; L^{2}\left(\mathbb{T}^{N}\right)\right)$ to the solution $\rho$ to the non-linear stochastic diffusion equation

$$
\mathrm{d} \rho-\operatorname{div}_{x}\left(\sigma(\rho)^{-1} K \nabla_{x} \rho\right) \mathrm{d} t=H \rho \mathrm{d} t+\rho Q^{\frac{1}{2}} \mathrm{~d} W_{t}, \text { in }[0, T] \times \mathbb{T}^{N},
$$

with initial condition $\rho(0)=\rho_{0}$ in $L^{2}\left(\mathbb{T}^{N}\right)$, and where $W$ is a cylindrical Wiener process on $L^{2}\left(\mathbb{T}^{N}\right)$,

$$
K:=\int_{V} a(v) \otimes a(v) F(v) \mathrm{d} \mu(v)
$$


and

$$
H:=\int_{E} n M^{-1} I(n) \mathrm{d} \nu(n) \in W^{1, \infty} .
$$

Remark The limit equation (2.13) can also be written in Stratonovich form

$$
\mathrm{d} \rho-\operatorname{div}_{x}\left(\sigma(\rho)^{-1} K \nabla_{x} \rho\right) \mathrm{d} t=\rho \circ Q^{\frac{1}{2}} \mathrm{~d} W_{t} .
$$

Notation In the sequel, we denote by $\lesssim$ the inequalities which are valid up to constants of the problem, namely $C_{*}, N, \sup _{\varepsilon>0}\left\|f_{0}^{\varepsilon}\right\|, \sup _{v \in V}|a(v)|, \sup _{v \in V} F(v), \sigma_{*}, \sigma^{*},\|\sigma\|_{\text {Lip }}$ and real constants.

\section{The generator}

The process $f^{\varepsilon}$ is not Markov (indeed, by (1.1), we need $m^{\varepsilon}$ to know the increments of $f^{\varepsilon}$ ) but the couple $\left(f^{\varepsilon}, m^{\varepsilon}\right)$ is. From now on, we denote by $\mathscr{L}^{\varepsilon}$ its infinitesimal generator, that is

$$
\mathscr{L}^{\varepsilon} \varphi(f, n):=\lim _{h \rightarrow 0} \frac{1}{h} \mathbb{E}\left[\varphi\left(f_{h}^{\varepsilon}, m_{h}^{\varepsilon}\right)-\varphi(f, n) \mid\left(f_{0}^{\varepsilon}, m_{0}^{\varepsilon}\right)=(f, n)\right],
$$

where $\varphi: L_{F^{-1}}^{2} \times E \rightarrow \mathbb{R}$ belongs to the domain of $\mathscr{L}^{\varepsilon}$. Thus we begin this section by introducing a special set of functions which lie in the domain of $\mathscr{L}^{\varepsilon}$ and satisfy the associated martingale problem.

In the following, if $\varphi: L_{F^{-1}}^{2} \rightarrow \mathbb{R}$ is differentiable with respect to $f \in L_{F^{-1}}^{2}$, we denote by $D \varphi(f)$ its differential at a point $f$ and we identify the differential with the gradient.

Definition 3.1. We say that $\varphi: L_{F^{-1}}^{2} \times E \rightarrow \mathbb{R}$ is a good test function if

(i) $(f, n) \mapsto \varphi(f, n)$ is differentiable with respect to $f$;

(ii) $(f, n) \mapsto D \varphi(f, n)$ is continuous from $L_{F^{-1}}^{2} \times E$ to $L_{F^{-1}}^{2}$ and maps bounded sets onto bounded sets;

(iii) for any $f \in L_{F^{-1}}^{2}, \varphi(f, \cdot) \in D_{M}$;

(iv) $(f, n) \mapsto M \varphi(f, n)$ is continuous from $L_{F^{-1}}^{2} \times E$ to $\mathbb{R}$ and maps bounded sets onto bounded sets.

Proposition 3.1. Let $\varphi$ be a good test function. Then, for all $(f, n) \in \mathrm{D}(A) \times E$,

$$
\mathscr{L}^{\varepsilon} \varphi(f, n)=-\frac{1}{\varepsilon}(A f, D \varphi(f))+\frac{1}{\varepsilon^{2}}(\sigma(\bar{f}) L f, D \varphi(f))+\frac{1}{\varepsilon}(f n, D \varphi(f))+\frac{1}{\varepsilon^{2}} M \varphi(f, n) .
$$

Furthermore, if $f_{0}^{\varepsilon} \in \mathrm{D}(A)$,

$$
M_{\varphi}^{\varepsilon}(t):=\varphi\left(f_{t}^{\varepsilon}, m_{t}^{\varepsilon}\right)-\varphi\left(f_{0}^{\varepsilon}, m_{0}^{\varepsilon}\right)-\int_{0}^{t} \mathscr{L}^{\varepsilon} \varphi\left(f_{s}^{\varepsilon}, m_{s}^{\varepsilon}\right) d s
$$

is a continuous and integrable $\left(\mathcal{F}_{t}^{\varepsilon}\right)_{t \geq 0}$ martingale, and if $|\varphi|^{2}$ is a good test function, its quadratic variation is given by

$$
\left\langle M_{\varphi}^{\varepsilon}\right\rangle_{t}=\int_{0}^{t}\left(\mathscr{L}^{\varepsilon}|\varphi|^{2}-2 \varphi \mathscr{L}^{\varepsilon} \varphi\right)\left(f_{s}^{\varepsilon}, m_{s}^{\varepsilon}\right) d s .
$$


Proof. We compute the expression of the infinitesimal generator as follows :

$$
\begin{aligned}
\mathscr{L}^{\varepsilon} \varphi(f, n) & =\lim _{h \rightarrow 0} \frac{1}{h} \mathbb{E}\left[\varphi\left(f_{h}^{\varepsilon}, m_{h}^{\varepsilon}\right)-\varphi(f, n) \mid\left(f_{0}^{\varepsilon}, m_{0}^{\varepsilon}\right)=(f, n)\right] \\
& =\lim _{h \rightarrow 0} \frac{1}{h} \mathbb{E}\left[\varphi\left(f_{h}^{\varepsilon}, m_{h}^{\varepsilon}\right)-\varphi\left(f, m_{h}^{\varepsilon}\right) \mid\left(f_{0}^{\varepsilon}, m_{0}^{\varepsilon}\right)=(f, n)\right] \\
& +\lim _{h \rightarrow 0} \frac{1}{h} \mathbb{E}\left[\varphi\left(f, m_{h}^{\varepsilon}\right)-\varphi(f, n) \mid m_{0}^{\varepsilon}=n\right]
\end{aligned}
$$

Since $\varphi$ verifies point $(i i i)$ of Definition 3.1, the second term of the last equality goes to $\varepsilon^{-2} M \varphi(f, n)$ when $h \rightarrow 0$. We now focus on the first term. With points $(i)-(i i)$ of Definition 3.1 , we have that $\varphi$ is continuously differentiable with respect to $f$. Thus

$$
\varphi\left(f_{h}^{\varepsilon}, m_{h}^{\varepsilon}\right)-\varphi\left(f, m_{h}^{\varepsilon}\right)=\int_{0}^{1} D \varphi\left(f+s\left(f_{h}^{\varepsilon}-f\right), m_{h}^{\varepsilon}\right)\left(f_{h}^{\varepsilon}-f\right) \mathrm{d} s .
$$

Besides, since $f_{0}^{\varepsilon}=f \in \mathrm{D}(A), f^{\varepsilon} \in C^{1}\left([0, T], L_{F^{-1}}^{2}\right)$ and we have

$$
f_{h}^{\varepsilon}-f=h \int_{0}^{1} \partial_{t} f_{u h}^{\varepsilon} \mathrm{d} u .
$$

Thus, we can rewrite the first term as

$$
\begin{aligned}
& =\lim _{h \rightarrow 0} \frac{1}{h} \mathbb{E}\left[\varphi\left(f_{h}^{\varepsilon}, m_{h}^{\varepsilon}\right)-\varphi\left(f, m_{h}^{\varepsilon}\right) \mid\left(f_{0}^{\varepsilon}, m_{0}^{\varepsilon}\right)=(f, n)\right] \\
& =\lim _{h \rightarrow 0} \mathbb{E}_{(f, n)}\left[\int_{0}^{1} \int_{0}^{1} a_{h}(w, s, u) \mathrm{d} u \mathrm{~d} s\right],
\end{aligned}
$$

with $a_{h}(w, s, u):=D \varphi\left(f+s\left(f_{h}^{\varepsilon}-f\right), m_{h}^{\varepsilon}\right)\left(\partial_{t} f_{u h}^{\varepsilon}\right)$ and where $\mathbb{E}_{(f, n)}$ denotes the expectation under the probability measure $\mathbb{P}_{(f, n)}:=\mathbb{P}\left(\cdot \mid\left(f_{0}^{\varepsilon}, m_{0}^{\varepsilon}\right)=(f, n)\right)$.

Recall that $D \varphi$ is continuous with respect to $(f, n)$ thanks to point (ii) of Definition 3.1, that $f^{\varepsilon}$ is $\mathbb{P}$-a.s. in $C^{1}\left([0, T], L_{F^{-1}}^{2}\right)$ and that $m^{\varepsilon}$ is stochastically continuous to conclude that $a_{h}$ converges in probability as $h \rightarrow 0$ to $D \varphi(f, n)\left(\partial_{t} f^{\varepsilon}(0)\right)$ in the probability space $\tilde{\Omega}:=$ $\left(\Omega \times[0,1] \times[0,1], \mathbb{P}_{(f, n)} \otimes \mathrm{d} x \otimes \mathrm{d} s\right)$. Furthermore, we prove that $\left(a_{h}\right)_{0 \leq h \leq 1}$ is uniformly integrable in $\tilde{\Omega}$ since it is uniformly bounded with respect to $0 \leq h \leq 1$ in $L^{\infty}(\tilde{\Omega})$. Indeed, with the fact that $L$ is a bounded operator, with (H1) and the fact that $\|n\|_{L^{\infty}\left(\mathbb{T}^{N}\right)} \lesssim 1$ for all $n \in E$, we get

$$
\left|a_{h}\right| \lesssim\left\|D \varphi\left(f+s\left(f_{h}^{\varepsilon}-f\right), m_{h}^{\varepsilon}\right)\right\|\left(\left\|f_{u h}^{\varepsilon}\right\|+\left\|A f_{u h}^{\varepsilon}\right\|\right) .
$$

With (2.12), we set

$$
R:=\sup _{t \in[0, T]}\left\|f_{t}^{\varepsilon}\right\|+\sup _{t \in[0, T]}\left\|A f_{t}^{\varepsilon}\right\| \in L^{\infty}(\Omega),
$$

and define $r:=\|R\|_{L^{\infty}(\Omega)}$. Then, since $D \varphi$ maps bounded sets on bounded sets, we can bound the term $\left\|D \varphi\left(f+s\left(f_{h}^{\varepsilon}-f\right), m_{h}^{\varepsilon}\right)\right\|$ by

$$
C:=\sup \left\{\|D \varphi(f, n)\|, f \in B_{L_{F^{-1}}^{2}}(0,\|f\|+r), n \in B_{E}\left(0, C_{*}\right)\right\} .
$$

So we are led to

$$
\left\|a_{h}\right\|_{L^{\infty}(\tilde{\Omega})} \lesssim C \cdot r
$$

which is what we announced. To prove the sequel of the proposition, we use the same kind of ideas and follow the proofs of [7, Proposition 6] and [9, Appendix 6.9]. 


\section{The limit generator}

In this section, we study the limit of the generator $\mathscr{L}^{\varepsilon}$ when $\varepsilon \rightarrow 0$. The limit generator $\mathscr{L}$ will characterize the limit stochastic fluid equation.

\subsection{Formal derivation of the corrections}

To derive the diffusive limiting equation, one has to study the limit as $\varepsilon$ goes to 0 of quantities of the form $\mathscr{L}^{\varepsilon} \varphi$ where $\varphi$ is a good test function. To do so, following the perturbed testfunctions method, we have to correct $\varphi$ so as to obtain a non-singular limit. We search the correction $\varphi^{\varepsilon}$ of $\varphi$ under the classical form:

$$
\varphi^{\varepsilon}:=\varphi+\varepsilon \varphi_{1}+\varepsilon^{2} \varphi_{2} .
$$

In this decomposition, $\varphi_{1}$ and $\varphi_{2}$ are respectively the first and second order corrections and are to be defined in the sequel so that

$$
\mathscr{L}^{\varepsilon} \varphi^{\varepsilon}=\mathscr{L} \varphi+O(\varepsilon)
$$

where $\mathscr{L}$ will be the limit generator. We restrict our study to smooth test-functions. Precisely, we introduce the set of spatial derivative operators up to order 3 :

$$
\mathcal{R}:=\left\{\partial_{i_{1}}^{e_{1}} \partial_{i_{2}}^{e_{2}} \partial_{i_{3}}^{e_{3}}, e \in\{0,1\}^{3}, i \in\{1, \ldots, N\}^{3},|i| \leq 3\right\}
$$

and we suppose that the test-function $\varphi$ is a good test, that $\varphi \in C^{3}\left(L_{F^{-1}}^{2}\right)$ and that there exists a constant $C_{\varphi}>0$ such that

$$
\left\{\begin{array}{l}
|\varphi(f)| \leq C_{\varphi}\left(1+\|f\|^{2}\right) \\
\|\Lambda D \varphi(f)\| \leq C_{\varphi}(1+\|f\|), \\
\left|D^{2} \varphi(f)\left(\Lambda_{1} h, \Lambda_{2} k\right)\right| \leq C_{\varphi}\|h\|\|k\|, \\
\left|D^{3} \varphi(f)\left(\Lambda_{1} h, \Lambda_{2} k, \Lambda_{3} l\right)\right| \leq C_{\varphi}\|h\|\|k\|\|l\|,
\end{array}\right.
$$

for any $f, h, k, l \in L_{F^{-1}}^{2}$ and $\Lambda, \Lambda_{1}, \Lambda_{2}, \Lambda_{3} \in \mathcal{R}$. Thanks to Proposition 3.1, and since $\varphi$ does not depend on $n \in E$, we can write

$$
\begin{aligned}
\mathscr{L}^{\varepsilon} \varphi^{\varepsilon}(f, n) & =-\frac{1}{\varepsilon}(A f, D \varphi(f))+\frac{1}{\varepsilon^{2}}(\sigma(\bar{f}) L f, D \varphi(f))+\frac{1}{\varepsilon}(f n, D \varphi(f)) \\
& -\left(A f, D \varphi_{1}(f)\right)+\frac{1}{\varepsilon}\left(\sigma(\bar{f}) L f, D \varphi_{1}(f)\right)+\left(f n, D \varphi_{1}(f)\right)+\frac{1}{\varepsilon} M \varphi_{1} \\
& -\varepsilon\left(A f, D \varphi_{2}(f)\right)+\left(\sigma(\bar{f}) L f, D \varphi_{2}(f)\right)+\varepsilon\left(f n, D \varphi_{2}(f)\right)+M \varphi_{2} .
\end{aligned}
$$

In the sequel, we do not care about the terms relative to the transport part $A$ of the equation since these terms will be handled as in the deterministic case (when $m^{\varepsilon} \equiv 0$ ). To be more precise, and as it will be shown in the sequel, the first term of (4.2) will give rise, as $\varepsilon$ goes to 0 , to the deterministic term in the limit generator $\mathscr{L}$ and the first terms of (4.3) and (4.4) are respectively of orders $\varepsilon$ and $\varepsilon^{2}$. For the remaining terms, in a first step, we would like to cancel those who have a singular power of $\varepsilon$. Thus we should impose that the two following equations hold:

$$
(\sigma(\bar{f}) L f, D \varphi(f))=0
$$




$$
\left(\sigma(\bar{f}) L f, D \varphi_{1}(f)\right)+M \varphi_{1}+(f n, D \varphi(f))=0 .
$$

Let us say a word about the fact that we chose to handle the terms relative to the transport part of the equation separately. When trying to correct these terms thanks to the correctors $\varphi_{1}$ and $\varphi_{2}$, the non-linearity $\sigma$ implies that the second corrector $\varphi_{2}$, unless we can write it formally, does not behave properly any more.

\subsubsection{Equation on $\varphi$}

Let us solve (4.5). We recall that $(g(t, f))_{t \geq 0}$ denotes the semigroup of the operator $\left.\sigma^{(}\right) L$. Equation (4.5) gives immediately that the map $t \mapsto \varphi(g(t, f))$ is constant. As a result, with $(2.5)$,

$$
\varphi(f)=\varphi(g(0, f))=\varphi(\varphi(g(\infty, f))=\varphi(\bar{f} F),
$$

so that $\varphi$ only depends on $\bar{f} F$. This implies, for all $h \in L_{F^{-1}}^{2}$,

$$
(h, D \varphi(f))=(\bar{h} F, D \varphi(\bar{f} F)) .
$$

\subsubsection{Equation on $\varphi_{1}$}

Next, we solve (4.6). We consider the Markov process $(g(t, f), m(t, n))_{t \geq 0}$. Its generator will be denoted by $\mathscr{M}$. We observe that equation (4.6) rewrites:

$$
\mathscr{M} \varphi_{1}(f, n)=-(f n, D \varphi(f)) .
$$

This Poisson equation will have a solution if the integral of $(f, n) \mapsto(f n, D \varphi(f))$ over $L_{F^{-1}}^{2} \times E$ equipped with the invariant measure of the process $(g(t, f), m(t, n))_{t \geq 0}$ is zero. So, we must verify that

$$
\int_{E}(\bar{f} F n, D \varphi(\bar{f} F)) \mathrm{d} \nu(n)=0,
$$

and this relation does hold since $m$ is centered. As a consequence, if we can prove the existence of the integral, we can write $\varphi_{1}$ as

$$
\varphi_{1}(f, n)=\int_{0}^{\infty} \mathbb{E}(g(t, f) m(t, n), D \varphi(g(t, f))) \mathrm{d} t .
$$

Then, we use (4.7), $\overline{g(t, f)}=\bar{f}$ and (2.7) and (2.8) to obtain

$$
\begin{aligned}
\varphi_{1}(f, n) & =\int_{0}^{\infty} \mathbb{E}(\bar{f} F m(t, n), D \varphi(\bar{f} F)) \mathrm{d} t=-\left(\bar{f} F M^{-1} I(n), D \varphi(\bar{f} F)\right) \\
& =-\left(f M^{-1} I(n), D \varphi(f)\right) .
\end{aligned}
$$

We are now able to state the

Proposition 4.1 (First corrector). Let $\varphi \in C^{3}\left(L_{F^{-1}}^{2}\right)$ be a good test-function satisfying (4.1) and depending only on $\bar{f} F$. For any $(f, n) \in L_{F^{-1}}^{2} \times E$, we define the first corrector $\varphi_{1}$ as

$$
\varphi_{1}(f, n):=-\left(f M^{-1} I(n), D \varphi(f)\right) .
$$

Furthermore, it satisfies the bounds

$$
\text { (i) }\left|\varphi_{1}(f, n)\right| \lesssim C_{\varphi}(1+\|f\|)^{2}, \quad(i i)\left\|A D \varphi_{1}(f, n)\right\| \lesssim C_{\varphi}(1+\|f\|) .
$$

Note that the bounds (4.8) are consequences of (2.10) and (4.1). 


\subsubsection{Equation on $\varphi_{2}$}

At this stage, we have

$$
\begin{aligned}
\mathscr{L}^{\varepsilon} \varphi^{\varepsilon}(f, n) & =-\frac{1}{\varepsilon}(A f, D \varphi(f))+\mathscr{M} \varphi_{2}+\left(f n, D \varphi_{1}(f)\right) \\
& -\left(A f, D \varphi_{1}(f)\right)-\varepsilon\left(A f, D \varphi_{2}(f)\right)+\varepsilon\left(f n, D \varphi_{2}(f)\right) .
\end{aligned}
$$

Note that the limit of $\mathscr{L}^{\varepsilon} \varphi^{\varepsilon}$ as $\varepsilon$ goes to 0 does depend on $n \in E$ with the term $\left(f n, D \varphi_{1}(f)\right)$. Since the expected limit is $\mathscr{L} \varphi$ where $\varphi$ does not depend on $n$, we have to correct this term to cancel the dependence with respect to $n$ of the limit. This is the aim of the second order correction $\varphi_{2}$. The right way to do so, given the mixing properties of the operator $\mathscr{M}$, is to subtract the mean value of this term under the invariant measure of the Markov process $(g(t, f), m(t, n))_{t \geq 0}$ governed by $\mathscr{M}$. We write

$$
\begin{aligned}
\mathscr{L}^{\varepsilon} \varphi^{\varepsilon}(f, n) & =-\frac{1}{\varepsilon}(A f, D \varphi(f))+\int_{E}\left(\bar{f} F n, D \varphi_{1}(\bar{f} F)\right) \mathrm{d} \nu(n) \\
& +\mathscr{M} \varphi_{2}+\left(f n, D \varphi_{1}(f)\right)-\int_{E}\left(\bar{f} F n, D \varphi_{1}(\bar{f} F)\right) \mathrm{d} \nu(n) \\
& -\left(A f, D \varphi_{1}(f)\right)-\varepsilon\left(A f, D \varphi_{2}(f)\right)+\varepsilon\left(f n, D \varphi_{2}(f)\right),
\end{aligned}
$$

and we can now define $\varphi_{2}$ as the solution of the well-posed Poisson equation

$$
\mathscr{M} \varphi_{2}=-\left(f n, D \varphi_{1}(f)\right)+\int_{E}\left(\bar{f} F n, D \varphi_{1}(\bar{f} F)\right) \mathrm{d} \nu(n) .
$$

Note that, thanks to the definition of $\varphi_{1}$ given above, we can compute

$$
\left(\bar{f} F n, D \varphi_{1}(\bar{f} F)\right)=-\left(f n M^{-1} I(n), D \varphi(f)\right)-D^{2} \varphi(f)\left(f M^{-1} I(n), f n\right)=: q(f, n)
$$

As a result, we easily have the following proposition.

Proposition 4.2 (Second corrector). Let $\varphi \in C^{3}\left(L_{F^{-1}}^{2}\right)$ be a good test-function satisfying (4.1) and depending only on $\bar{f} F$. For any $(f, n) \in L_{F^{-1}}^{2} \times E$, we define the second corrector $\varphi_{2}$ as

$$
\varphi_{2}(f, n):=\mathbb{E} \int_{0}^{\infty}\left(\int_{E}(q(\bar{f} F, n) \mathrm{d} \nu(n)-q(g(t, f), m(t, n))) \mathrm{d} t,\right.
$$

which is well defined and satisfies the bounds

$$
\text { (i) }\left|\varphi_{2}(f, n)\right| \lesssim C_{\varphi}(1+\|f\|)^{2}, \quad \text { (ii) }\left\|A D \varphi_{2}(f, n)\right\| \lesssim C_{\varphi}(1+\|f\|) .
$$

The existence of $\varphi_{2}$ is based on (2.9) and the bounds (4.10) are proved using (2.10) and (4.1).

\subsubsection{Summary}

The correctors $\varphi_{1}$ and $\varphi_{2}$ being defined as above in Propositions 4.1 and 4.2, we are finally led to

$$
\begin{aligned}
\mathscr{L}^{\varepsilon} \varphi^{\varepsilon}(f, n) & =-\frac{1}{\varepsilon}(A f, D \varphi(f))+\int_{E}\left(\bar{f} F n, D \varphi_{1}(\bar{f} F)\right) \mathrm{d} \nu(n) \\
& -\left(A f, D \varphi_{1}(f)\right)-\varepsilon\left(A f, D \varphi_{2}(f)\right)+\varepsilon\left(f n, D \varphi_{2}(f)\right) .
\end{aligned}
$$


We are now able to define the limit generator $\mathscr{L}$ as, for all $\rho \in L^{2}\left(\mathbb{T}^{N}\right)$,

$$
\begin{aligned}
\mathscr{L} \varphi(\rho):=\left(\operatorname{div}_{x}\left(\sigma(\rho)^{-1} K \nabla_{x} \rho\right) F, D \varphi(\rho F)\right) & -\int_{E}\left(\rho F n M^{-1} I(n), D \varphi(\rho F)\right) \mathrm{d} \nu(n) \\
& -\int_{E} D^{2} \varphi(\rho F)\left(\rho F M^{-1} I(n), \rho F n\right) \mathrm{d} \nu(n),
\end{aligned}
$$

and we have shown the following equality

$$
\begin{aligned}
\mathscr{L}^{\varepsilon} \varphi^{\varepsilon}(f, n) & =\mathscr{L} \varphi(\bar{f})-\frac{1}{\varepsilon}(A f, D \varphi(f))-\left(\operatorname{div}_{x}\left(\sigma(\bar{f})^{-1} K \nabla_{x} \bar{f}\right) F, D \varphi(\bar{f} F)\right) \\
& -\left(A f, D \varphi_{1}(f)\right)-\varepsilon\left(A f, D \varphi_{2}(f)\right)+\varepsilon\left(f n, D \varphi_{2}(f)\right) .
\end{aligned}
$$

\section{$5 \quad$ Uniform bound in $L_{F^{-1}}^{2}$}

In this section, we prove a uniform estimate of the $L_{F^{-1}}^{2}$ norm of the solution $f^{\varepsilon}$ with respect to $\varepsilon$. To do so, we will again use the perturbed test functions method. The result is the following:

Proposition 5.1. Let $p \geq 1$ and $f_{0}^{\varepsilon} \in \mathrm{D}(A)$. We have the two following bounds

$$
\begin{gathered}
\mathbb{E} \sup _{t \in[0, T]}\left\|f_{t}^{\varepsilon}\right\|^{p} \lesssim 1, \\
\mathbb{E}\left(\int_{0}^{T}\left\|\sigma^{\frac{1}{2}}\left(\overline{f_{s}^{\varepsilon}}\right) L f_{s}^{\varepsilon}\right\|^{2} \mathrm{~d} s\right)^{p} \lesssim \varepsilon^{2 p} .
\end{gathered}
$$

Proof. We set, for all $f \in L_{F^{-1}}^{2}, \varphi(f):=\frac{1}{2}\|f\|^{2}$, which is easily seen to be a good test function. Then, with Proposition 3.1, the fact that $A$ is skew-adjoint, (2.4), and the fact that $\varphi$ does not depend on $n \in E$, we get for $f \in \mathrm{D}(A)$ and $n \in E$,

$$
\begin{aligned}
\mathscr{L}^{\varepsilon} \varphi(f, n) & =-\frac{1}{\varepsilon}(A f, f)+\frac{1}{\varepsilon^{2}}(\sigma(\bar{f}) L f, f)+\frac{1}{\varepsilon}(f n, f)+\frac{1}{\varepsilon^{2}} M \varphi(f, n) \\
& =-\frac{1}{\varepsilon^{2}}\left\|\sigma^{\frac{1}{2}}(\bar{f}) L f\right\|^{2}+\frac{1}{\varepsilon}(f n, f) .
\end{aligned}
$$

The first term has a favourable behaviour for our purpose. The second term is more difficult to control and we correct $\varphi$ thanks to the perturbed test-functions method to get rid of it: we recall the formal computations done in Section 4.1 and we set $\varphi_{1}(f, n)=-\left(f, M^{-1} I(n) f\right)$ and $\varphi^{\varepsilon}:=\varphi(f, n)+\varepsilon \varphi_{1}$. We can show that $\varphi_{1}$ is a good test function with, thanks to Proposition 3.1 ,

$$
\begin{aligned}
\varepsilon \mathscr{L}^{\varepsilon} \varphi_{1}(f, n)=- & \frac{2}{\varepsilon}\left(\sigma(\bar{f}) L f, M^{-1} I(n) f\right)-2\left(A f, M^{-1} I(n) f\right) \\
& -2\left(f n, M^{-1} I(n) f\right)-\frac{1}{\varepsilon}(f n, f) .
\end{aligned}
$$

As a consequence, we are led to

$$
\begin{aligned}
\mathscr{L}^{\varepsilon} \varphi^{\varepsilon}(f, n)=- & \frac{1}{\varepsilon^{2}}\left\|\sigma^{\frac{1}{2}}(\bar{f}) L f\right\|^{2}-\frac{2}{\varepsilon}\left(\sigma(\bar{f}) L f, M^{-1} I(n) f\right)-2\left(A f, M^{-1} I(n) f\right) \\
& -2\left(f n, M^{-1} I(n) f\right) .
\end{aligned}
$$


We use (2.10) and the hypothesis (H1) made on $\sigma$ to bound the second term:

$$
\begin{aligned}
\frac{2}{\varepsilon}\left(\sigma(\bar{f}) L f, M^{-1} I(n) f\right) & \leq 2 C_{*}\left(\sigma^{*}\right)^{\frac{1}{2}} \varepsilon^{-1}\left\|\sigma^{\frac{1}{2}}(\bar{f}) L f\right\|\|f\| \\
& \leq \frac{1}{2 \varepsilon^{2}}\left\|\sigma^{\frac{1}{2}}(\bar{f}) L f\right\|^{2}+2 C_{*}^{2} \sigma^{*}\|f\|^{2} .
\end{aligned}
$$

Furthermore, for the last two terms, we write

$$
\begin{aligned}
-2\left(A f, M^{-1} I(n) f\right)-2\left(f n, M^{-1} I(n) f\right) & =\left(f^{2}, A M^{-1} I(n)\right)-2\left(f n, M^{-1} I(n) f\right) \\
& \leq\|f\|^{2}\|a\|_{L^{\infty}(V)} C_{*}+2 C_{*}^{2}\|f\|^{2} .
\end{aligned}
$$

To sum up, we have proved that

$$
\mathscr{L}^{\varepsilon} \varphi^{\varepsilon}(f, n) \lesssim-\frac{1}{2 \varepsilon^{2}}\left\|\sigma^{\frac{1}{2}}(\bar{f}) L f\right\|^{2}+\|f\|^{2} .
$$

As in Proposition 3.1, since $\varphi^{\varepsilon}$ is a good test function, we now define

$$
M^{\varepsilon}(t):=\varphi^{\varepsilon}\left(f_{t}^{\varepsilon}, m_{t}^{\varepsilon}\right)-\varphi^{\varepsilon}\left(f_{0}^{\varepsilon}, m_{0}^{\varepsilon}\right)-\int_{0}^{t} \mathscr{L}^{\varepsilon} \varphi^{\varepsilon}\left(f_{s}^{\varepsilon}, m_{s}^{\varepsilon}\right) \mathrm{d} s,
$$

which is a continuous and integrable $\left(\mathcal{F}_{t}^{\varepsilon}\right)_{t \geq 0}$ martingale. By definition of $\varphi, \varphi^{\varepsilon}$ and $M^{\varepsilon}$, we obtain

$$
\frac{1}{2}\left\|f_{t}^{\varepsilon}\right\|^{2}=\frac{1}{2}\left\|f_{0}^{\varepsilon}\right\|^{2}-\varepsilon\left(\varphi_{1}\left(f_{t}^{\varepsilon}, m_{t}^{\varepsilon}\right)-\varphi_{1}\left(f_{0}^{\varepsilon}, m_{0}^{\varepsilon}\right)\right)+\int_{0}^{t} \mathscr{L}^{\varepsilon} \varphi^{\varepsilon}\left(f_{s}^{\varepsilon}, m_{s}^{\varepsilon}\right) \mathrm{d} s+M^{\varepsilon}(t) .
$$

Since we have obviously $\left|\varphi_{1}(f, n)\right| \lesssim\|f\|^{2}$, we can write, with (5.3),

$$
\left\|f_{t}^{\varepsilon}\right\|^{2} \lesssim\left\|f_{0}^{\varepsilon}\right\|^{2}+\varepsilon\left\|f_{t}^{\varepsilon}\right\|+\int_{0}^{t}-\frac{1}{2 \varepsilon^{2}}\left\|\sigma^{\frac{1}{2}}\left(\overline{f_{s}^{\varepsilon}}\right) L f_{s}^{\varepsilon}\right\|^{2}+\left\|f_{s}^{\varepsilon}\right\|^{2} \mathrm{~d} s+\sup _{t \in[0, T]}\left|M^{\varepsilon}(t)\right|,
$$

i.e. for $\varepsilon$ sufficiently small,

$$
\int_{0}^{t} \frac{1}{2 \varepsilon^{2}}\left\|\sigma^{\frac{1}{2}}\left(\overline{f_{s}^{\varepsilon}}\right) L f_{s}^{\varepsilon}\right\|^{2} \mathrm{~d} s+\left\|f_{t}^{\varepsilon}\right\|^{2} \lesssim\left\|f_{0}^{\varepsilon}\right\|^{2}+\int_{0}^{t}\left\|f_{s}^{\varepsilon}\right\|^{2} d s+\sup _{t \in[0, T]}\left|M^{\varepsilon}(t)\right|,
$$

and by Gronwall lemma,

$$
\int_{0}^{t} \frac{1}{2 \varepsilon^{2}}\left\|\sigma^{\frac{1}{2}}\left(\overline{f_{s}^{\varepsilon}}\right) L f_{s}^{\varepsilon}\right\|^{2} \mathrm{~d} s+\left\|f_{t}^{\varepsilon}\right\|^{2} \lesssim\left\|f_{0}^{\varepsilon}\right\|^{2}+\sup _{t \in[0, T]}\left|M^{\varepsilon}(t)\right| .
$$

Note that $\left|\varphi^{\varepsilon}\right|^{2}$ is a good test function with, thanks to (2.10) and (2.11),

$$
\left.\left|\mathscr{L}^{\varepsilon}\right| \varphi^{\varepsilon}\right|^{2}-2 \varphi^{\varepsilon} \mathscr{L}^{\varepsilon} \varphi^{\varepsilon}|=| M\left|\varphi_{1}\right|^{2}-2 \varphi_{1} M \varphi_{1} \mid \lesssim\|f\|^{4},
$$

and that, with Proposition 3.1, the quadratic variation of $M^{\varepsilon}(t)$ is given by

$$
\left\langle M^{\varepsilon}\right\rangle_{t}=\int_{0}^{t}\left(\mathscr{L}^{\varepsilon}\left|\varphi^{\varepsilon}\right|^{2}-2 \varphi^{\varepsilon} \mathscr{L}^{\varepsilon} \varphi^{\varepsilon}\right)\left(f_{s}^{\varepsilon}, m_{s}^{\varepsilon}\right) \mathrm{d} s .
$$


As a result, with Burkholder-Davis-Gundy and Hölder inequalities, we get

$$
\mathbb{E} \sup _{t \in[0, T]}\left|M^{\varepsilon}(t)\right|^{p} \lesssim \mathbb{E}\left|\left\langle M^{\varepsilon}\right\rangle_{T}\right|^{\frac{p}{2}} \lesssim \int_{0}^{T} \mathbb{E}\left\|f_{s}^{\varepsilon}\right\|^{2 p} \mathrm{~d} s
$$

Neglecting the first (positive) term of the left-hand side in (5.4), we have

$$
\mathbb{E}\left\|f_{t}^{\varepsilon}\right\|^{2 p} \lesssim \mathbb{E}\left\|f_{0}^{\varepsilon}\right\|^{2 p}+\mathbb{E} \sup _{t \in[0, T]}\left|M^{\varepsilon}(t)\right|^{p},
$$

so that we get

$$
\mathbb{E}\left\|f_{T}^{\varepsilon}\right\|^{2 p} \lesssim \mathbb{E}\left\|f_{0}^{\varepsilon}\right\|^{2 p}+\int_{0}^{T} \mathbb{E}\left\|f_{s}^{\varepsilon}\right\|^{2 p} \mathrm{~d} s
$$

and, by Gronwall lemma,

$$
\mathbb{E}\left\|f_{T}^{\varepsilon}\right\|^{2 p} \lesssim \mathbb{E}\left\|f_{0}^{\varepsilon}\right\|^{2 p}
$$

This actually holds true for any $t \in[0, T]$. Thus, using (5.5) and (5.6) in (5.4) finally gives the expected bounds.

Remark We define $g^{\varepsilon}:=f^{\varepsilon}-\rho^{\varepsilon} F=-L f^{\varepsilon}$. Since we have $\sigma \geq \sigma_{*}$, the bound (5.2) gives that, for all $p \geq 1$,

$$
\left(\varepsilon^{-1} g^{\varepsilon}\right)_{\varepsilon>0} \text { is bounded in } L^{p}\left(\Omega ; L^{2}\left(0, T ; L_{F^{-1}}^{2}\right)\right) .
$$

In the sequel, we must deal with the non-linear term. To do so, we need some compactness in the space variable of the process $\left(\rho^{\varepsilon}\right)_{\varepsilon>0}$. The following proposition is a first step to this purpose.

Proposition 5.2. We assume that hypothesis (2.3) is satisfied. Let $p \geq 1$ and $s \in(0, \theta / 2)$. We have the bound

$$
\mathbb{E}\left(\int_{0}^{T}\left\|\rho_{s}^{\varepsilon}\right\|_{H^{s}\left(\mathbb{T}^{N}\right)}^{2} \mathrm{~d} s\right)^{p} \lesssim 1
$$

Proof. Note that with $\sigma \leq \sigma^{*}$, the remark (5.7) and equation (1.1), we observe that

$$
\left(\varepsilon \partial_{t} f^{\varepsilon}+a(v) \cdot \nabla_{x} f^{\varepsilon}-f^{\varepsilon} m^{\varepsilon}\right)_{\varepsilon>0} \text { is bounded in } L^{p}\left(\Omega ; L^{2}\left(0, T ; L_{F^{-1}}^{2}\right)\right) .
$$

Furthermore, $\left(f^{\varepsilon}\right)_{\varepsilon>0}$ is bounded in $L^{p}\left(\Omega ; L^{2}\left(0, T ; L_{F^{-1}}^{2}\right)\right)$ with (5.1) and $\left|m^{\varepsilon}\right| \leq C_{*}$ so that

$$
\left(\varepsilon \partial_{t} f^{\varepsilon}+a(v) \cdot \nabla_{x} f^{\varepsilon}\right)_{\varepsilon>0} \text { is bounded in } L^{p}\left(\Omega ; L^{2}\left(0, T ; L_{F^{-1}}^{2}\right)\right) .
$$

Then, thanks to (2.3), we apply an averaging lemma to conclude. Precisely, [10, Theorem 3.1] in the unstationary case applies a.s. with $\beta=\gamma=0, p_{1}=q_{1}=p_{2}=q_{2}=2, a=0, k=\theta$ and

$$
f=f^{\varepsilon}, \quad g=\varepsilon \partial_{t} f^{\varepsilon}+a(v) \cdot \nabla_{x} f^{\varepsilon},
$$

and gives the bound

$$
\left\|\rho^{\varepsilon}\right\|_{B_{\infty}^{\frac{\theta}{2}, 2} \leq} \leq C\left\|f^{\varepsilon}\right\|^{\frac{1}{2}}\left\|\varepsilon \partial_{t} f^{\varepsilon}+a(v) \cdot \nabla_{x} f^{\varepsilon}\right\|^{\frac{1}{2}} \quad \text { a.s. }
$$

Since, for any $s<\theta / 2, H^{s} \subset B_{\infty, \infty}^{\frac{\theta}{2}}$, it yields, for $p \geq 1$,

$$
\mathbb{E}\left(\int_{0}^{T}\left\|\rho_{s}^{\varepsilon}\right\|_{H^{s}}^{2} \mathrm{~d} s\right)^{p} \leq C \mathbb{E}\left(\int_{0}^{T}\left\|f_{s}^{\varepsilon}\right\|\left\|\varepsilon \partial_{t} f_{s}^{\varepsilon}+a(v) \cdot \nabla_{x} f_{s}^{\varepsilon}\right\| \mathrm{d} s\right)^{p},
$$

so that the result follows with Cauchy Schwarz inequality and (5.1) and (5.9). This concludes the proof. 


\section{Tightness}

We want to prove the convergence in law of the family $\left(\rho^{\varepsilon}\right)_{\varepsilon>0}$ : in this section, we study the tightness of the processes $\left(\rho^{\varepsilon}\right)_{\varepsilon>0}$ in the space $C\left([0, T], H^{-\eta}\left(\mathbb{T}^{N}\right)\right)$ where $\eta>0$. In fact, this will not be sufficient to pass to the limit in the non-linear term. As a consequence, we also prove that $\left(\rho^{\varepsilon}\right)_{\varepsilon>0}$ is tight in the space $L^{2}\left(0, T ; L^{2}\left(\mathbb{T}^{N}\right)\right)$.

Proposition 6.1. Let $\eta>0$. Then the sequence $\left(\rho^{\varepsilon}\right)_{\varepsilon>0}$ is tight in the spaces $C\left([0, T], H^{-\eta}\left(\mathbb{T}^{N}\right)\right)$ and $L^{2}\left(0, T ; L^{2}\left(\mathbb{T}^{N}\right)\right)$.

Proof. Step 1: control of the modulus of continuity of $\rho^{\varepsilon}$ in $H^{-\eta}\left(\mathbb{T}^{N}\right)$. Let $\eta>0$ be fixed. For any $\delta>0$, we define

$$
w(\rho, \delta):=\sup _{|t-s|<\delta}\|\rho(t)-\rho(s)\|_{H^{-\eta}\left(\mathbb{T}^{N}\right)}
$$

the modulus of continuity of a function $\rho \in C\left([0, T], H^{-\eta}\left(\mathbb{T}^{N}\right)\right)$. In this first step of the proof, we want to obtain the following bound

$$
\mathbb{E} w\left(\rho^{\varepsilon}, \delta\right) \lesssim \varepsilon+\delta^{\tau},
$$

for some positive $\tau$. To do so, we use the perturbed test-functions method. Let $\left(p_{j}\right)_{j \in \mathbb{N}^{N}}$ the Fourier orthonormal basis of $L^{2}\left(\mathbb{T}^{N}\right)$ and $J$ the operator

$$
J:=\left(\mathrm{I}-\Delta_{x}\right)^{-\frac{1}{2}} .
$$

Let $j \in \mathbb{N}^{N}$. We set

$$
\varphi_{j}(f):=\left(f, p_{j} F\right), \quad f \in L_{F^{-1}}^{2},
$$

and we define the first order corrections by, see Section 4.1,

$$
\varphi_{1, j}(f, n):=-\left(f M^{-1} I(n), p_{j} F\right), \quad(f, n) \in L_{F^{-1}}^{2} \times E .
$$

We finally define $\varphi_{j}^{\varepsilon}:=\varphi_{j}+\varepsilon \varphi_{1, j}$, which is easily seen to be a good test-function, so that, thanks to Proposition 3.1, we consider the continuous martingales

$$
M_{j}^{\varepsilon}(t):=\varphi_{j}^{\varepsilon}\left(f_{t}^{\varepsilon}, m_{t}^{\varepsilon}\right)-\varphi_{j}^{\varepsilon}\left(f_{0}^{\varepsilon}, m_{0}^{\varepsilon}\right)-\int_{0}^{t} \mathscr{L}^{\varepsilon} \varphi_{j}^{\varepsilon}\left(f_{s}^{\varepsilon}, m_{s}^{\varepsilon}\right) \mathrm{d} s .
$$

We also define,

$$
\theta_{j}^{\varepsilon}(t):=\varphi_{j}\left(f_{0}^{\varepsilon}\right)+\int_{0}^{t} \mathscr{L}^{\varepsilon} \varphi_{j}^{\varepsilon}\left(f_{s}^{\varepsilon}, m_{s}^{\varepsilon}\right) \mathrm{d} s+M_{j}^{\varepsilon}(t)
$$

Note that

$$
\theta_{j}^{\varepsilon}(t)=\varphi_{j}\left(f_{t}^{\varepsilon}\right)+\varepsilon\left(\varphi_{1, j}\left(f_{t}^{\varepsilon}, m_{t}^{\varepsilon}\right)-\varphi_{1, j}\left(f_{0}^{\varepsilon}, m_{0}^{\varepsilon}\right)\right),
$$

so that, with the definitions of $\varphi_{j}$ and $\varphi_{1, j}$, Cauchy-Schwarz inequality, we easily get

$$
\left|\theta_{j}^{\varepsilon}(t)\right| \lesssim \sup _{t \in[0, T]}\left\|f^{\varepsilon}(t)\right\|\left\|p_{j}\right\|_{L_{x}^{2}}=\sup _{t \in[0, T]}\left\|f^{\varepsilon}(t)\right\| .
$$

Hence, by the uniform $L_{F^{-1}}^{2}$ bound (5.1),

$$
\mathbb{E} \sup _{t \in[0, T]}\left|\theta_{j}^{\varepsilon}(t)\right| \lesssim 1
$$


With (6.2) and the uniform $L_{F^{-1}}^{2}$ bound (5.1), we also deduce

$$
\mathbb{E} \sup _{t \in[0, T]}\left|\varphi_{j}\left(\rho_{t}^{\varepsilon}\right)-\theta_{j}^{\varepsilon}(t)\right| \lesssim \varepsilon
$$

From now on, we fix $\gamma>N / 2+2$ and we remark that, by (6.3), a.s. and for all $t \in[0, T]$, the series defined by $u_{t}^{\varepsilon}:=\sum_{j \in \mathbb{N}^{N}} \theta_{j}^{\varepsilon}(t) J^{\gamma} p_{j}$ converges in $L^{2}\left(\mathbb{T}^{N}\right)$. We then set

$$
\theta^{\varepsilon}(t):=J^{-\gamma} \sum_{j \in \mathbb{N}^{N}} \theta_{j}^{\varepsilon}(t) J^{\gamma} p_{j}
$$

which exists a.s. and for all $t \in[0, T]$ in $H^{-\gamma}\left(\mathbb{T}^{N}\right)$. And with (6.4), we obtain

$$
\mathbb{E} \sup _{t \in[0, T]}\left\|\rho^{\varepsilon}(t)-\theta^{\varepsilon}(t)\right\|_{H^{-\gamma}\left(\mathbb{T}^{N}\right)} \lesssim \varepsilon
$$

Actually, by interpolation, the continuous embedding $L^{2}\left(\mathbb{T}^{N}\right) \subset H^{-\eta}\left(\mathbb{T}^{N}\right)$ and the uniform $L_{F^{-1}}^{2}$ bound (5.1), we have

$$
\mathbb{E} \sup _{|t-s|<\delta}\|\rho(t)-\rho(s)\|_{H^{-\eta^{b}}} \leq \mathbb{E} \sup _{|t-s|<\delta}\|\rho(t)-\rho(s)\|_{H^{-\eta^{\sharp}}}^{v}
$$

for a certain $v>0$ if $\eta^{\sharp}>\eta^{b}>0$. As a result, it is indeed sufficient to work with $\eta=\gamma$. In view of (6.5), we first want to obtain an estimate of the increments of $\theta^{\varepsilon}$. We have, for $j \in \mathbb{N}^{N}$ and $0 \leq s \leq t \leq T$,

$$
\theta_{j}^{\varepsilon}(t)-\theta_{j}^{\varepsilon}(s)=\int_{s}^{t} \mathscr{L}^{\varepsilon} \varphi_{j}^{\varepsilon}\left(f_{\sigma}^{\varepsilon}, m_{\sigma}^{\varepsilon}\right) \mathrm{d} \sigma+M_{j}^{\varepsilon}(t)-M_{j}^{\varepsilon}(s) .
$$

We then control the two terms on the right-hand side of (6.6). Let us begin with the first one. Note that, since $D \varphi_{j}(f) \equiv p_{j} F$ and $D \varphi_{1, j}(f) \equiv-M^{-1} I(n) p_{j} F$, we obtain thanks to (4.9) with $\varphi_{2} \equiv 0$,

$$
\mathscr{L}^{\varepsilon} \varphi_{j}^{\varepsilon}\left(f_{\sigma}^{\varepsilon}, m_{\sigma}^{\varepsilon}\right)=-\frac{1}{\varepsilon}\left(A f_{\sigma}^{\varepsilon}, p_{j} F\right)+\left(A f_{\sigma}^{\varepsilon}, M^{-1} I\left(m_{\sigma}^{\varepsilon}\right) p_{j} F\right)-\left(f_{\sigma}^{\varepsilon} m_{\sigma}^{\varepsilon}, M^{-1} I\left(m_{\sigma}^{\varepsilon}\right) p_{j} F\right) .
$$

Since, with (2.2), we have $\overline{a(v) f_{\sigma}^{\varepsilon}}=\overline{a(v) g_{\sigma}^{\varepsilon}}$ where $g^{\varepsilon}$ has been defined previously as $g^{\varepsilon}:=$ $f^{\varepsilon}-\rho^{\varepsilon} F$, we can write

$$
\left(A f_{\sigma}^{\varepsilon}, p_{j} F\right)=\int_{\mathbb{T}^{N}} \operatorname{div}_{x}\left(\overline{a(v) f_{\sigma}^{\varepsilon}}\right) p_{j} \mathrm{~d} x=\int_{\mathbb{T}^{N}} \operatorname{div}_{x}\left(\overline{a(v) g_{\sigma}^{\varepsilon}}\right) p_{j} \mathrm{~d} x=\left(A g_{\sigma}^{\varepsilon}, p_{j} F\right)
$$

and, as a consequence, since $a$ is bounded, we are led to

$$
\frac{1}{\varepsilon}\left(A f_{\sigma}^{\varepsilon}, p_{j} F\right) \lesssim\left\|\varepsilon^{-1} g_{\sigma}^{\varepsilon}\right\|\left\|\nabla_{x} p_{j}\right\|_{L^{2}}
$$

Similarly, we can show that

$$
\left(A f_{\sigma}^{\varepsilon}, M^{-1} I\left(m_{\sigma}^{\varepsilon}\right) p_{j} F\right) \lesssim\left\|g_{\sigma}^{\varepsilon}\right\|\left(1+\left\|\nabla_{x} p_{j}\right\|_{L^{2}}\right) .
$$

Since we have obviously $\left(f_{\sigma}^{\varepsilon} m_{\sigma}^{\varepsilon}, M^{-1} I\left(m_{\sigma}^{\varepsilon}\right) p_{j} F\right) \lesssim\left\|f_{\sigma}^{\varepsilon}\right\|$, we can conclude that

$$
\left|\mathscr{L}^{\varepsilon} \varphi_{j}^{\varepsilon}\left(f_{\sigma}^{\varepsilon}, m_{\sigma}^{\varepsilon}\right)\right| \lesssim C_{j}\left[\left\|\varepsilon^{-1} g_{\sigma}^{\varepsilon}\right\|+\left\|g_{\sigma}^{\varepsilon}\right\|+\left\|f_{\sigma}^{\varepsilon}\right\|\right]
$$


where $C_{j}:=1+\left\|\nabla_{x} p_{j}\right\|_{L^{2}} \leq 1+|j|$. Thanks to (5.1) and (5.7) with $p=4$, we have that $\left(\varepsilon^{-1} g^{\varepsilon}\right)_{\varepsilon>0},\left(g^{\varepsilon}\right)_{\varepsilon>0}$ and $\left(f^{\varepsilon}\right)_{\varepsilon>0}$ are bounded in $L^{4}\left(\Omega ; L^{2}\left(0, T ; L_{F^{-1}}^{2}\right)\right)$. As a consequence, (6.7) and an application of Hölder's inequality gives

$$
\mathbb{E}\left|\int_{s}^{t} \mathscr{L}^{\varepsilon} \varphi_{j}^{\varepsilon}\left(f_{\sigma}^{\varepsilon}, m_{\sigma}^{\varepsilon}\right) d \sigma\right|^{4} \lesssim C_{j}^{4}|t-s|^{2}
$$

Furthermore, using Burkholder-Davis-Gundy inequality, we can control the second term of the right-hand side of (6.6) as

$$
\mathbb{E}\left|M_{j}^{\varepsilon}(t)-M_{j}^{\varepsilon}(s)\right|^{4} \lesssim \mathbb{E}\left|\left\langle M_{j}^{\varepsilon}\right\rangle_{t}-\left\langle M_{j}^{\varepsilon}\right\rangle_{s}\right|^{2},
$$

where the quadratic variation $\left\langle M_{j}^{\varepsilon}\right\rangle$ is given by

$$
\left\langle M_{j}^{\varepsilon}\right\rangle_{t}=\int_{0}^{t}\left(M\left|\varphi_{1, j}\right|^{2}-2 \varphi_{1, j} M \varphi_{1, j}\right)\left(f_{s}^{\varepsilon}, m_{s}^{\varepsilon}\right) \mathrm{d} s .
$$

With the definition of $\varphi_{1, j},(2.10),(2.11)$ and the uniform $L_{F^{-1}}^{2}$ bound (5.1), it is now easy to get

$$
\mathbb{E}\left|M_{j}^{\varepsilon}(t)-M_{j}^{\varepsilon}(s)\right|^{4} \lesssim|t-s|^{2} .
$$

Finally we have $\mathbb{E}\left|\theta_{j}^{\varepsilon}(t)-\theta_{j}^{\varepsilon}(s)\right|^{4} \lesssim\left(1+|j|^{4}\right)|t-s|^{2}$. Since we took $\gamma>N / 2+2$, we can conclude that

$$
\mathbb{E}\left\|\theta^{\varepsilon}(t)-\theta^{\varepsilon}(s)\right\|_{H^{-\gamma}\left(\mathbb{T}^{N}\right)}^{4} \lesssim|t-s|^{2} .
$$

It easily follows that, for $v<1 / 2$,

$$
\mathbb{E}\left\|\theta^{\varepsilon}\right\|_{W^{v, 4}\left(0, T, H^{-\gamma}\left(\mathbb{T}^{N}\right)\right)}^{4} \lesssim 1
$$

and by the embedding

$$
W^{v, 4}\left(0, T, H^{-\gamma}\left(\mathbb{T}^{N}\right)\right) \subset \mathcal{C}^{\tau}\left(0, T, H^{-\gamma}\left(\mathbb{T}^{N}\right)\right), \quad \tau<v-\frac{1}{4},
$$

we obtain that $\mathbb{E} w\left(\theta^{\varepsilon}, \delta\right) \lesssim \delta^{\tau}$ for a certain positive $\tau$. Finally, with (6.5), we can now conclude the first step of the proof since

$$
\mathbb{E} w\left(\rho^{\varepsilon}, \delta\right) \leq 2 \mathbb{E} \sup _{t \in[0, T]}\left\|\rho_{t}^{\varepsilon}-\theta_{t}^{\varepsilon}\right\|_{H^{-\gamma}\left(\mathbb{T}^{N}\right)}+\mathbb{E} w\left(\theta^{\varepsilon}, \delta\right) \lesssim \varepsilon+\delta^{\tau} .
$$

Step 2: tightness in $C\left([0, T] ; H^{-\eta}\left(\mathbb{T}^{N}\right)\right)$. Since the embedding $L^{2}\left(\mathbb{T}^{N}\right) \subset H^{-\eta}\left(\mathbb{T}^{N}\right)$ is compact, and by Ascoli's Theorem, the set

$$
K_{R}:=\left\{\rho \in C\left([0, T], H^{-\eta}\left(\mathbb{T}^{N}\right)\right), \sup _{t \in[0, T]}\|\rho\|_{L^{2}\left(\mathbb{T}^{N}\right)} \leq R, w(\rho, \delta)<\varepsilon(\delta)\right\},
$$

where $R>0$ and $\varepsilon(\delta) \rightarrow 0$ when $\delta \rightarrow 0$, is compact in $C\left([0, T], H^{-\eta}\left(\mathbb{T}^{N}\right)\right)$. By Prokohrov's Theorem, the tightness of $\left(\rho^{\varepsilon}\right)_{\varepsilon>0}$ in $C\left([0, T], H^{-\eta}\left(\mathbb{T}^{N}\right)\right)$ will follow if we prove that for all $\sigma>0$, there exists $R>0$ such that

$$
\mathbb{P}\left(\sup _{t \in[0, T]}\left\|\rho^{\varepsilon}\right\|_{L^{2}\left(\mathbb{T}^{N}\right)}>R\right)<\sigma
$$


and

$$
\lim _{\delta \rightarrow 0} \limsup _{\varepsilon \rightarrow 0} \mathbb{P}\left(w\left(\rho^{\varepsilon}, \delta\right)>\sigma\right)=0 .
$$

With Markov's inequality and the uniform $L_{F^{-1}}^{2}$ bound (5.1), we have

$$
\mathbb{P}\left(\sup _{t \in[0, T]}\left\|\rho^{\varepsilon}\right\|_{L^{2}\left(\mathbb{T}^{N}\right)}>R\right) \leq \mathbb{P}\left(\sup _{t \in[0, T]}\left\|f^{\varepsilon}\right\|>R\right) \lesssim R^{-1},
$$

which gives (6.9). And we deduce (6.10) by Markov's inequality and the bound (6.1) since

$$
\begin{aligned}
\lim _{\delta \rightarrow 0} \limsup _{\varepsilon \rightarrow 0} \mathbb{P}\left(w\left(\rho^{\varepsilon}, \delta\right)>\sigma\right) & \leq \lim _{\delta \rightarrow 0} \limsup _{\varepsilon \rightarrow 0} \sigma^{-1} \mathbb{E} w\left(\rho^{\varepsilon}, \delta\right) \\
& \lesssim \lim _{\delta \rightarrow 0} \limsup _{\varepsilon \rightarrow 0} \sigma^{-1}\left(\varepsilon+\delta^{\tau}\right)=0 .
\end{aligned}
$$

Step 3: tightness in $L^{2}\left(0, T ; L^{2}\left(\mathbb{T}^{N}\right)\right)$. Similarly, due to [12, Theorem 5], the set

$$
K_{R}:=\left\{\rho \in L^{2}\left(0, T ; L^{2}\left(\mathbb{T}^{N}\right)\right), \int_{0}^{T}\left\|\rho_{t}\right\|_{H^{s}\left(\mathbb{T}^{N}\right)}^{2} \mathrm{~d} t \leq R, w(\rho, \delta)<\varepsilon(\delta)\right\},
$$

where $R>0, s>0$ and $\varepsilon(\delta) \rightarrow 0$ when $\delta \rightarrow 0$, is compact in $L^{2}\left(0, T ; L^{2}\left(\mathbb{T}^{N}\right)\right)$. By Prokhorov's Theorem, the tightness of $\left(\rho^{\varepsilon}\right)_{\varepsilon>0}$ in $L^{2}\left(0, T ; L^{2}\left(\mathbb{T}^{N}\right)\right)$ will follow if we prove that for all $\sigma>0$, there exists $R>0$ such that

$$
\mathbb{P}\left(\int_{0}^{T}\left\|\rho_{t}\right\|_{H^{s}\left(\mathbb{T}^{N}\right)}^{2} \mathrm{~d} t>R\right)<\sigma
$$

and

$$
\lim _{\delta \rightarrow 0} \limsup _{\varepsilon \rightarrow 0} \mathbb{P}\left(w\left(\rho^{\varepsilon}, \delta\right)>\sigma\right)=0 .
$$

But (6.11) and (6.12) are consequences of Markov's inequality and the bounds (5.8) with $p=1$ and (6.1) so that the proof is complete.

\section{Convergence}

We conclude here the proof of Theorem 2.2. The idea is now, by the tightness result and Prokhorov Theorem, to take a subsequence of $\left(\rho^{\varepsilon}\right)_{\varepsilon>0}$ that converges in law to some probability measure. Then we show that this limiting probability is actually uniquely determined by the limit generator $\mathscr{L}$ defined above.

We fix $\eta>0$. By Proposition 6.1 and Prokhorov's Theorem, there is a subsequence of $\left(\rho^{\varepsilon}\right)_{\varepsilon>0}$, still denoted $\left(\rho^{\varepsilon}\right)_{\varepsilon>0}$, and a probability measure $P$ on the spaces $C\left([0, T], H^{-\eta}\right)$ and $L^{2}\left(0, T ; L^{2}\right)$ such that

$$
P^{\varepsilon} \rightarrow P \text { weakly in } C\left([0, T], H^{-\eta}\right) \text { and } L^{2}\left(0, T ; L^{2}\right),
$$

where $P^{\varepsilon}$ stands for the law of $\rho^{\varepsilon}$. We now identify the probability measure $P$.

Since the spaces $C\left([0, T], H^{-\eta}\right)$ and $L^{2}\left(0, T ; L^{2}\right)$ are separable, we can apply Skohorod representation Theorem $[3]$, so that there exists a new probability space $(\widetilde{\Omega}, \widetilde{\mathcal{F}}, \widetilde{\mathbb{P}})$ and random variables

$$
\widetilde{\rho^{\varepsilon}}, \widetilde{\rho}: \widetilde{\Omega} \rightarrow C\left([0, T], H^{-\eta}\right) \cap L^{2}\left(0, T ; L^{2}\right),
$$


with respective law $P^{\varepsilon}$ and $P$ such that $\widetilde{\rho^{\varepsilon}} \rightarrow \widetilde{\rho}$ in $C\left([0, T], H^{-\eta}\right)$ and $L^{2}\left(0, T ; L^{2}\right) \widetilde{\mathbb{P}}-$ a.s. In the sequel, for the sake of clarity, we do not write any more the tildes.

Note that, with (5.7), we can also suppose that $\varepsilon^{-1} g^{\varepsilon}$ converges to some $g$ weakly in the space $L^{2}\left(\Omega ; L^{2}\left(0, T ; L_{F^{-1}}^{2}\right)\right)$. Similarly, with $(2.10)$, we assume that $m^{\varepsilon}$ converges to $m$ weakly in $L^{2}\left(\Omega ; L^{2}\left(0, T ; L_{F^{-1}}^{2}\right)\right)$. Before going on the proof, we want to identify the weak limit $g$ of $\varepsilon^{-1} g^{\varepsilon}$.

Lemma 7.1. In $L^{2}\left(\Omega ; L^{2}\left(0, T ; L^{2}\right)\right)$, we have the relation

$$
\overline{a(v) g}=-\sigma(\rho)^{-1} K \nabla_{x} \rho .
$$

Proof. We define $D_{T}:=(0, T) \times \mathbb{T}^{N}$. Since $f^{\varepsilon}$ satisfies equation (1.1), we can write, for any $\psi \in C_{c}^{\infty}\left(D_{T}\right)$ and $\theta \in L^{\infty}\left(V \times \Omega ; \mathbb{R}^{N}\right)$,

$$
\begin{aligned}
\mathbb{E} \int_{D_{T} \times V} f^{\varepsilon} F^{-1}\left(-\varepsilon \partial_{t} \psi-a \cdot \nabla_{x} \psi\right) \theta & =\mathbb{E} \int_{D_{T} \times V} \frac{1}{\varepsilon} \sigma\left(\overline{f^{\varepsilon}}\right) L f^{\varepsilon} F^{-1} \psi \theta \\
& +\mathbb{E} \int_{D_{T} \times V} m^{\varepsilon} f^{\varepsilon} F^{-1} \psi \theta .
\end{aligned}
$$

We recall that we set $g^{\varepsilon}:=f^{\varepsilon}-\rho^{\varepsilon} F$ and that $L f^{\varepsilon}=L g^{\varepsilon}$ so that we have

$$
\begin{aligned}
& \mathbb{E} \int_{D_{T} \times V}-\varepsilon f^{\varepsilon} F^{-1} \partial_{t} \psi \theta-\rho^{\varepsilon} a \cdot \nabla_{x} \psi \theta-g^{\varepsilon} F^{-1} a \cdot \nabla_{x} \psi \theta \\
& \quad=\mathbb{E} \int_{D_{T} \times V} \sigma\left(\rho^{\varepsilon}\right) L\left(\varepsilon^{-1} g^{\varepsilon}\right) F^{-1} \psi \theta+\mathbb{E} \int_{D_{T} \times V} m^{\varepsilon} f^{\varepsilon} F^{-1} \psi \theta .
\end{aligned}
$$

Since $\left(f^{\varepsilon}\right)_{\varepsilon>0}$ and $\left(\varepsilon^{-1} g^{\varepsilon}\right)_{\varepsilon>0}$ are bounded in $L^{2}\left(\Omega ; L^{2}\left(0, T ; L_{F^{-1}}^{2}\right)\right)$ by (5.1) and (5.7), and with the $\mathbb{P}$-a.s. convergence $\rho^{\varepsilon} \rightarrow \rho$ in $L^{2}\left(0, T ; L_{F^{-1}}^{2}\right)$ coupled with the uniform integrability of the family $\left(\rho^{\varepsilon}\right)_{\varepsilon>0}$ obtained with (5.1), we have that the left-hand side of the previous equality actually converges as $\varepsilon \rightarrow 0$ to

$$
\mathbb{E} \int_{D_{T} \times V}-\rho a \cdot \nabla_{x} \psi \theta .
$$

Note that, $\mathbb{P}$-a.s., we have the following convergences in $L^{2}\left(0, T ; L_{F^{-1}}^{2}\right)$

$$
\sigma\left(\rho^{\varepsilon}\right) \rightarrow \sigma(\rho), \quad L\left(\varepsilon^{-1} g^{\varepsilon}\right) \rightarrow L g, \quad f^{\varepsilon} \rightarrow \rho F, \quad m^{\varepsilon} \rightarrow \mathrm{m},
$$

where the first convergence is justified by the Lipschitz continuity of $\sigma$. As a result, since all the quantities above are uniformly integrable with respect to $\varepsilon$ thanks to (5.1), (5.7) and (2.10), the right-hand side of the previous equality converges as $\varepsilon \rightarrow 0$ to

$$
\mathbb{E} \int_{D_{T} \times V} \sigma(\rho) L(g) F^{-1} \psi \theta+\mathbb{E} \int_{D_{T} \times V} \operatorname{m} \rho \psi \theta .
$$

Thus, we have

$$
\mathbb{E} \int_{D_{T} \times V}-\rho a \cdot \nabla_{x} \psi \theta=\mathbb{E} \int_{D_{T} \times V} \sigma(\rho) L(g) F^{-1} \psi \theta+\mathbb{E} \int_{D_{T} \times V} \mathrm{~m} \rho \psi \theta .
$$


Let $\xi$ be an arbitrary bounded measurable function on $\Omega$. We now set $\theta(v, \omega)=a(v) F(v) \xi(\omega)$; note that we do have $\theta \in L^{\infty}\left(V \times \Omega, \mathbb{R}^{N}\right)$. With (2.2) and the relation $L g=\bar{g} F-g$, we obtain

$$
-\mathbb{E} \int_{D_{T} \times V} \rho a \cdot \nabla_{x} \psi a F=-\mathbb{E} \int_{D_{T} \times V} \sigma(\rho) g a(v) \psi .
$$

Since this relation holds for any $\xi \in L^{\infty}(\Omega)$ and $\psi \in C_{c}^{\infty}\left(D_{T}\right)$, we deduce that $\nabla_{x} \rho \in$ $L^{2}\left(\Omega, L^{2}\left(D_{T}\right)\right)$ and that

$$
\overline{a(v) g}=-\sigma(\rho)^{-1} K \nabla_{x} \rho,
$$

and this concludes the proof.

Let $\varphi \in C^{3}\left(L_{F^{-1}}^{2}\right)$ a good test-function satisfying (4.1). We define $\varphi^{\varepsilon}$ as in Section 4.1. Since $\varphi^{\varepsilon}$ is a good test-function, we have that

$$
\varphi^{\varepsilon}\left(f_{t}^{\varepsilon}, m_{t}^{\varepsilon}\right)-\varphi^{\varepsilon}\left(f_{0}^{\varepsilon}, m_{0}^{\varepsilon}\right)-\int_{0}^{t} \mathscr{L}^{\varepsilon} \varphi^{\varepsilon}\left(f_{s}^{\varepsilon}, m_{s}^{\varepsilon}\right) \mathrm{d} s, \quad t \in[0, T],
$$

is a continuous martingale for the filtration generated by $\left(f_{s}^{\varepsilon}\right)_{s \in[0, T]}$. As a result, if $\Psi$ denotes a continuous and bounded function from $L^{2}\left(\mathbb{T}^{N}\right)^{n}$ to $\mathbb{R}$, we have

$$
\mathbb{E}\left[\left(\varphi^{\varepsilon}\left(f_{t}^{\varepsilon}, m_{t}^{\varepsilon}\right)-\varphi^{\varepsilon}\left(f_{s}^{\varepsilon}, m_{s}^{\varepsilon}\right)-\int_{s}^{t} \mathscr{L}^{\varepsilon} \varphi^{\varepsilon}\left(f_{u}^{\varepsilon}, m_{u}^{\varepsilon}\right) \mathrm{d} u\right) \Psi\left(\rho_{s_{1}}^{\varepsilon}, \ldots, \rho_{s_{n}}^{\varepsilon}\right)\right]=0,
$$

for any $0 \leq s_{1} \leq \ldots \leq s_{n} \leq s \leq t$. Our final purpose is to pass to the limit $\varepsilon \rightarrow 0$ in (7.1). In the sequel, we assume that the function $\varphi$ and $\Psi$ are also continuous on the space $H^{-\eta}$, which is always possible with an approximation argument: it suffices to consider $\varphi_{r}:=\varphi\left(\left(\mathrm{I}-r \Delta_{x}\right)^{-\frac{\eta}{2}}\right.$. $)$ and $\Psi_{r}:=\Psi\left(\left(\mathrm{I}-r \Delta_{x}\right)^{-\frac{\eta}{2}} \cdot, \ldots,\left(\mathrm{I}-r \Delta_{x}\right)^{-\frac{\eta}{2}} \cdot\right)$ as $r \rightarrow 0$. With (4.12), we divide the left-hand side of (7.1) in four parts. Precisely, we define, for $i \in\{1, \ldots, 4\}$

$$
\begin{aligned}
\tau_{1}^{\varepsilon} & :=\varphi^{\varepsilon}\left(f_{t}^{\varepsilon}, m_{t}^{\varepsilon}\right)-\varphi^{\varepsilon}\left(f_{s}^{\varepsilon}, m_{s}^{\varepsilon}\right), \\
\tau_{2}^{\varepsilon} & :=\int_{s}^{t} \mathscr{L} \varphi\left(\rho_{u}^{\varepsilon}\right) \mathrm{d} u \\
\tau_{3}^{\varepsilon} & :=\int_{s}^{t}-\frac{1}{\varepsilon}\left(A f_{u}^{\varepsilon}, D \varphi\left(f_{u}^{\varepsilon}\right)\right)-\left(\operatorname{div}_{x}\left(\sigma\left(\rho_{u}^{\varepsilon}\right)^{-1} K \nabla_{x} \rho_{u}^{\varepsilon}\right) F, D \varphi\left(\rho_{u}^{\varepsilon} F\right)\right) \mathrm{d} u, \\
\tau_{4}^{\varepsilon} & :=\int_{s}^{t}-\left(A f_{u}^{\varepsilon}, D \varphi_{1}\left(f_{u}^{\varepsilon}\right)\right)-\varepsilon\left(A f_{u}^{\varepsilon}, D \varphi_{2}\left(f_{u}^{\varepsilon}\right)\right)+\varepsilon\left(f_{u}^{\varepsilon} m_{u}^{\varepsilon}, D \varphi_{2}\left(f_{u}^{\varepsilon}\right)\right) \mathrm{d} u .
\end{aligned}
$$

Study of $\tau_{1}^{\varepsilon}$. We recall that $\varphi^{\varepsilon}\left(f_{t}^{\varepsilon}, m_{t}^{\varepsilon}\right)=\varphi\left(\rho_{t}^{\varepsilon} F\right)+\varepsilon \varphi_{1}\left(f_{t}^{\varepsilon}, m_{t}^{\varepsilon}\right)+\varepsilon^{2} \varphi_{2}\left(f_{t}^{\varepsilon}, m_{t}^{\varepsilon}\right)$ so that, with the $\mathbb{P}$-a.s. convergence of $\rho^{\varepsilon}$ to $\rho$ in $C\left([0, T], H^{-\eta}\right)$ and the bounds $(i)$ of (4.8) and (4.10), we have that $\tau_{1}^{\varepsilon}$ converges $\mathbb{P}$-a.s. to $\varphi\left(\rho_{t} F\right)-\varphi\left(\rho_{s} F\right)$ as $\varepsilon$ goes to 0 . Furthermore, with the continuity of $\Psi$ in $H^{-\eta}$, we also have that $\Psi\left(\rho_{s_{1}}^{\varepsilon}, \ldots, \rho_{s_{n}}^{\varepsilon}\right)$ converges $\mathbb{P}$-a.s. to $\Psi\left(\rho_{s_{1}}, \ldots, \rho_{s_{n}}\right)$. Finally, since the family $\tau_{1}^{\varepsilon} \Psi\left(\rho_{s_{1}}^{\varepsilon}, \ldots, \rho_{s_{n}}^{\varepsilon}\right)$ is uniformly integrable with respect to $\varepsilon$ thanks to (4.1), the bounds (i) of (4.8) and (4.10) and the uniform $L_{F^{-1}}^{2}$ bound (5.1), we have that

$$
\mathbb{E}\left[\tau_{1}^{\varepsilon} \Psi\left(\rho_{s_{1}}^{\varepsilon}, \ldots, \rho_{s_{n}}^{\varepsilon}\right)\right] \rightarrow \mathbb{E}\left[\left(\varphi\left(\rho_{t} F\right)-\varphi\left(\rho_{s} F\right)\right) \Psi\left(\rho_{s_{1}}, \ldots, \rho_{s_{n}}\right)\right] .
$$


Study of $\tau_{2}^{\varepsilon}$. We recall, with (4.11), that

$$
\begin{array}{r}
\mathscr{L} \varphi\left(\rho_{u}^{\varepsilon}\right)=\left(\operatorname{div}_{x}\left(\sigma\left(\rho_{u}^{\varepsilon}\right)^{-1} K \nabla_{x} \rho_{u}^{\varepsilon}\right) F, D \varphi\left(\rho_{u}^{\varepsilon} F\right)\right)-\int_{E}\left(\rho_{u}^{\varepsilon} F n M^{-1} I(n), D \varphi\left(\rho_{u}^{\varepsilon} F\right)\right) d \nu(n) \\
-\int_{E} D^{2} \varphi\left(\rho_{u}^{\varepsilon} F\right)\left(\rho_{u}^{\varepsilon} F M^{-1} I(n), \rho_{u}^{\varepsilon} F n\right) d \nu(n) .
\end{array}
$$

Thanks to the $\mathbb{P}$-a.s. convergence of $\rho^{\varepsilon}$ to $\rho$ in $L^{2}\left(0, T ; L^{2}\right)$ and with $\varphi \in C^{3}\left(L_{F^{-1}}^{2}\right)$, we can pass to the limit $\varepsilon \rightarrow 0$ in the term

$$
\int_{s}^{t} \int_{E}-\left(\rho_{u}^{\varepsilon} F n M^{-1} I(n), D \varphi\left(\rho_{u}^{\varepsilon} F\right)\right)-D^{2} \varphi\left(\rho_{u}^{\varepsilon} F\right)\left(\rho_{u}^{\varepsilon} F M^{-1} I(n), \rho_{u}^{\varepsilon} F n\right) d \nu(n) \mathrm{d} u .
$$

Regarding the first term of $\mathscr{L} \varphi\left(\rho_{u}^{\varepsilon}\right)$, we introduce

$$
G(\rho):=\int_{0}^{\rho} \frac{\mathrm{d} y}{\sigma(y)}
$$

which is, thanks to the hypothesis (H1) made on $\sigma$, Lipschitz continuous on $L^{2}\left(\mathbb{T}^{N}\right)$. Now the first term of $\mathscr{L} \varphi\left(\rho_{u}^{\varepsilon}\right)$ writes

$$
\left(\operatorname{div}_{x}\left(\sigma\left(\rho_{u}^{\varepsilon}\right)^{-1} K \nabla_{x} \rho_{u}^{\varepsilon}\right) F, D \varphi\left(\rho_{u}^{\varepsilon} F\right)\right)=\left(\operatorname{div}_{x} \nabla_{x} G\left(\rho_{u}^{\varepsilon}\right) F, D \varphi\left(\rho_{u}^{\varepsilon} F\right)\right) .
$$

Furthermore, with (4.1), the mapping $\rho \mapsto \partial_{x_{i}, x_{j}}^{2} D \varphi(\rho F)$ is continuous on $L^{2}\left(\mathbb{T}^{N}\right)$. As a result, we can now pass to the limit in the term

$$
\int_{s}^{t}\left(\operatorname{div}_{x}\left(\sigma\left(\rho_{u}^{\varepsilon}\right)^{-1} K \nabla_{x} \rho_{u}^{\varepsilon}\right) F, D \varphi\left(\rho_{u}^{\varepsilon} F\right)\right) \mathrm{d} u .
$$

To sum up, we obtain that $\tau_{2}^{\varepsilon}$ converges $\mathbb{P}$-a.s. to $\int_{s}^{t} \mathscr{L} \varphi\left(\rho_{u}\right) \mathrm{d} u$ as $\varepsilon$ goes to 0 . Finally, since the family $\tau_{2}^{\varepsilon} \Psi\left(\rho_{s_{1}}^{\varepsilon}, \ldots, \rho_{s_{n}}^{\varepsilon}\right)$ is uniformly integrable with respect to $\varepsilon$ thanks to (4.1) and the uniform $L_{F^{-1}}^{2}$ bound (5.1), we have that

$$
\mathbb{E}\left[\tau_{2}^{\varepsilon} \Psi\left(\rho_{s_{1}}^{\varepsilon}, \ldots, \rho_{s_{n}}^{\varepsilon}\right)\right] \rightarrow \mathbb{E}\left[\left(\int_{s}^{t} \mathscr{L} \varphi\left(\rho_{u}\right) \mathrm{d} u\right) \Psi\left(\rho_{s_{1}}, \ldots, \rho_{s_{n}}\right)\right] .
$$

Study of $\tau_{3}^{\varepsilon}$. First of all, we observe that, with the decomposition $f^{\varepsilon}=\rho^{\varepsilon} F+g^{\varepsilon}$, (4.7) and $(2.2)$,

$$
-\varepsilon^{-1}\left(A f_{u}^{\varepsilon}, D \varphi\left(f_{u}^{\varepsilon}\right)\right)=-\varepsilon^{-1}\left(A g_{u}^{\varepsilon}, D \varphi\left(f_{u}^{\varepsilon}\right)\right),
$$

so that, with the $\mathbb{P}$-a.s. convergences in $L^{2}\left(0, T ; L^{2}\right)$

$$
\varepsilon^{-1} g^{\varepsilon} \rightarrow g, \quad \rho^{\varepsilon} \rightarrow \rho
$$

and the continuity of the mapping $\rho \mapsto A D \varphi(\rho F)$ thanks to (4.1), we obtain that the first term of $\tau_{3}^{\varepsilon}$ converges $\mathbb{P}-$ a.s. to

$$
-\int_{s}^{t}\left(\overline{A g_{u}} F, D \varphi\left(\rho_{u} F\right)\right) \mathrm{d} u
$$


And, with Lemma 7.1, this term writes

$$
\int_{s}^{t}\left(\operatorname{div}_{x}\left(\sigma\left(\rho_{u}\right)^{-1} K \nabla_{x} \rho_{u}\right) F, D \varphi\left(\rho_{u} F\right)\right) \mathrm{d} u .
$$

Furthermore, similarly as the case of $\tau_{2}^{\varepsilon}$, we have that the second term of $\tau_{3}^{\varepsilon}$ converges $\mathbb{P}$-a.s. to the opposite of (7.2). As a result, $\tau_{3}^{\varepsilon}$ converges $\mathbb{P}$-a.s. to 0 . Finally, since the family $\tau_{3}^{\varepsilon} \Psi\left(\rho_{s_{1}}^{\varepsilon}, \ldots, \rho_{s_{n}}^{\varepsilon}\right)$ is uniformly integrable with respect to $\varepsilon$ thanks to (4.1), the uniform $L_{F^{-1}}^{2}$ bound (5.1) and the bound (5.7) on $\left(\varepsilon^{-1} g^{\varepsilon}\right)_{\varepsilon>0}$, we have that

$$
\mathbb{E}\left[\tau_{3}^{\varepsilon} \Psi\left(\rho_{s_{1}}^{\varepsilon}, \ldots, \rho_{s_{n}}^{\varepsilon}\right)\right] \rightarrow 0 .
$$

Study of $\tau_{4}^{\varepsilon}$. If we transform the two first terms of $\tau_{4}^{\varepsilon}$ exactly as we do for the first term of $\tau_{3}^{\varepsilon}$, it is then easy, using the uniform bounds (5.1) and (5.7) and the bounds (ii) of (4.8) and (4.10), to get

$$
\mathbb{E}\left[\tau_{4}^{\varepsilon} \Psi\left(\rho_{s_{1}}^{\varepsilon}, \ldots, \rho_{s_{n}}^{\varepsilon}\right)\right]=O(\varepsilon)
$$

To sum up, we can pass to the limit $\varepsilon \rightarrow 0$ in (7.1) to obtain

$$
\mathbb{E}\left[\left(\varphi\left(\rho_{t} F\right)-\varphi\left(\rho_{s} F\right)-\int_{s}^{t} \mathscr{L} \varphi\left(\rho_{u}\right) \mathrm{d} u\right) \Psi\left(\rho_{s_{1}}, \ldots, \rho_{s_{n}}\right)\right]=0 .
$$

We recall that this is valid for all $n \in \mathbb{N}, 0 \leq s_{1} \leq \ldots \leq s_{n} \leq s \leq t \in[0, T]$ and all $\Psi$ continuous and bounded function on $L^{2}\left(\mathbb{T}^{N}\right)^{n}$. Now, let $\xi$ be a smooth function on $L^{2}\left(\mathbb{T}^{N}\right)$. We choose $\varphi(f)=(f, \xi F)$. We can easily verify that $\varphi$ and $|\varphi|^{2}$ belong to $C^{3}\left(L_{F^{-1}}^{2}\right)$ and that they are good test-function satisfying (4.1). Thus, we obtain that

$$
\begin{aligned}
& N_{t}:=\varphi\left(\rho_{t} F\right)-\varphi\left(\rho_{0} F\right)-\int_{0}^{t} \mathscr{L} \varphi\left(\rho_{u}\right) \mathrm{d} u, \quad t \in[0, T], \\
& |\varphi|^{2}\left(\rho_{t} F\right)-|\varphi|^{2}\left(\rho_{0} F\right)-\int_{0}^{t} \mathscr{L}|\varphi|^{2}\left(\rho_{u}\right) \mathrm{d} u, \quad t \in[0, T],
\end{aligned}
$$

are continuous martingales with respect to the filtration generated by $\left(\rho_{s}\right)_{s \in[0, T]}$. It implies (see appendix 6.9 in [9]) that the quadratic variation of $N$ is given by

$$
\langle N\rangle_{t}=\int_{0}^{t}\left[\mathscr{L}|\varphi|^{2}\left(\rho_{u}\right)-2 \varphi\left(\rho_{u}\right) \mathscr{L} \varphi\left(\rho_{u}\right)\right] \mathrm{d} u, \quad t \in[0, T] .
$$

Furthermore, we have

$$
\begin{aligned}
\mathscr{L}|\varphi|^{2}\left(\rho_{u}\right)-2 \varphi\left(\rho_{u}\right) \mathscr{L} \varphi\left(\rho_{u}\right) & =-2 \int_{E}\left(\rho_{u} F n, \xi F\right)\left(\rho_{u} F M^{-1} I(n), \xi F\right) \mathrm{d} \nu(n) \\
& =2 \mathbb{E} \int_{0}^{\infty}\left(\rho_{u} F m_{0}, \xi F\right)\left(\rho_{u} F m_{t}, \xi F\right) \mathrm{d} t \\
& =\mathbb{E} \int_{\mathbb{R}}\left(\rho_{u} F m_{0}, \xi F\right)\left(\rho_{u} F m_{t}, \xi F\right) \mathrm{d} t \\
& =\int_{\mathbb{T}^{N}} \int_{\mathbb{T}^{N}} \rho_{u}(x) \xi(x) \rho_{u}(y) \xi(y) k(x, y) \mathrm{d} x \mathrm{~d} y \\
& =\left\|\rho_{u} Q^{\frac{1}{2}} \xi\right\|_{L^{2}}^{2} .
\end{aligned}
$$


This is valid for all smooth function $\xi$ of $L^{2}\left(\mathbb{T}^{N}\right)$ so we deduce that

$$
M_{t}:=\rho_{t}-\rho_{0}-\int_{0}^{t} \operatorname{div}_{x}\left(\sigma\left(\rho_{s}\right)^{-1} K \nabla_{x} \rho_{s}\right) \mathrm{d} s-\int_{0}^{t} \rho_{s} H \mathrm{~d} s, \quad t \in[0, T],
$$

is a martingale with quadratic variation

$$
\int_{0}^{t} \rho_{s} Q^{\frac{1}{2}}\left(\rho_{s} Q^{\frac{1}{2}}\right)^{*} \mathrm{~d} s .
$$

Thanks to martingale representation Theorem, see [5, Theorem 8.2], up to a change of probability space, there exists a cylindrical Wiener process $W$ such that

$$
\rho_{t}-\rho_{0}-\int_{0}^{t} \operatorname{div}_{x}\left(\sigma\left(\rho_{s}\right)^{-1} K \nabla_{x} \rho_{s}\right) \mathrm{d} s-\int_{0}^{t} \rho_{s} H \mathrm{~d} s=\int_{0}^{t} \rho_{s} Q^{\frac{1}{2}} \mathrm{~d} W_{s}, \quad t \in[0, T] .
$$

This gives that $\rho$ has the law of a weak solution to the equation (2.13) with paths in $C\left([0, T], H^{-\eta}\right) \cap$ $L^{2}\left(0, T ; L^{2}\right)$. Since this equation has a unique solution with paths in the space $C\left([0, T], H^{-\eta}\right) \cap$ $L^{2}\left(0, T ; L^{2}\right)$, and since pathwise uniqueness implies uniqueness in law, we deduce that $P$ is the law of this solution and is uniquely determined. Finally, by the uniqueness of the limit, the whole sequence $\left(P^{\varepsilon}\right)_{\varepsilon>0}$ converges to $P$ weakly in the spaces of probability measures on $C\left([0, T], H^{-\eta}\right)$ and $L^{2}\left(0, T ; L^{2}\right)$. This concludes the proof of Theorem 2.2.

\section{References}

[1] C. Bardos, F. Golse, and B. Perthame. The Rosseland approximation for the radiative transfer equations. Comm. Pure Appl. Math., 40(6):691-721, 1987.

[2] C. Bardos, F. Golse, B. Perthame, and R. Sentis. The nonaccretive radiative transfer equations: existence of solutions and Rosseland approximation. J. Funct. Anal., 77(2):434$460,1988$.

[3] P. Billingsley. Convergence of Probability Measures. Wiley Series in Probability and Statistics. John Wiley \& Sons, 2009.

[4] T. Cazenave and A. Haraux. An introduction to semilinear evolution equations. Oxford Lecture Series in Mathematics and its Applications. Clarendon Press, 1998.

[5] G. Da Prato and J. Zabczyk. Stochastic Equations in Infinite Dimensions. Encyclopedia of Mathematics and Its Applications. Cambridge University Press, 2008.

[6] A. de Bouard and M. Gazeau. A diffusion approximation theorem for a nonlinear PDE with application to random birefringent optical fibers. Ann. Appl. Probab., 22(6):2460$2504,2012$.

[7] A. Debussche and J. Vovelle. Diffusion limit for a stochastic kinetic problem. Commun. Pure Appl. Anal., 11(6):2305-2326, 2012.

[8] P. Degond, T. Goudon, and F. Poupaud. Diffusion limit for nonhomogeneous and nonmicro-reversible processes. Indiana Univ. Math. J., 49(3):1175-1198, 2000. 
[9] J.P. Fouque, J. Garnier, G. Papanicolaou, and K. Solna. Wave Propagation and Time Reversal in Randomly Layered Media. Stochastic Modelling and Applied Probability. Springer, 2010.

[10] P.-E. Jabin. Averaging lemmas and dispersion estimates for kinetic equations. Riv. Mat. Univ. Parma (8), 1:71-138, 2009.

[11] G. C. Papanicolaou, D. Stroock, and S. R. S. Varadhan. Martingale approach to some limit theorems. Duke Univ. Math. Ser. Duke Univ., 1977.

[12] J. Simon. Compact sets in the space $L^{p}(0, T ; B)$. Ann. Mat. Pura Appl. (4), 146:65-96, 1987. 